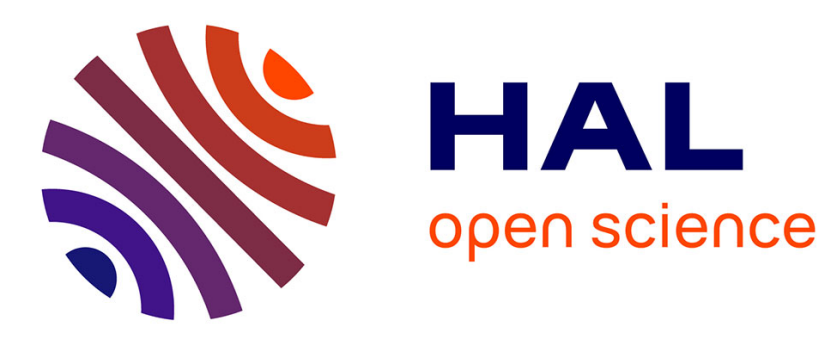

\title{
Structural estimation of farmers' risk and ambiguity preferences: a field experiment
}

Douadia Bougherara, Xavier Gassmann, Laurent Piet, Arnaud Reynaud

\section{To cite this version:}

Douadia Bougherara, Xavier Gassmann, Laurent Piet, Arnaud Reynaud. Structural estimation of farmers' risk and ambiguity preferences: a field experiment. European Review of Agricultural Economics, 2017, à paraître (5), à paraître. hal-01607282

\section{HAL Id: hal-01607282 \\ https://hal.science/hal-01607282}

Submitted on 26 May 2020

HAL is a multi-disciplinary open access archive for the deposit and dissemination of scientific research documents, whether they are published or not. The documents may come from teaching and research institutions in France or abroad, or from public or private research centers.
L'archive ouverte pluridisciplinaire $\mathbf{H A L}$, est destinée au dépôt et à la diffusion de documents scientifiques de niveau recherche, publiés ou non, émanant des établissements d'enseignement et de recherche français ou étrangers, des laboratoires publics ou privés.

$$
\text { Copyright }
$$




\title{
Structural estimation of farmers' risk and ambiguity preferences: A field experiment
}

\author{
April 19, 2017
}

\begin{abstract}
Ever since Ellsberg (1961), the distinction between risk, where agents assign well-defined probabilities to possible outcomes, and ambiguity, where agents do not, has been of particular interest. Using a carefully-designed field experiment, we elicit information about risk and ambiguity preferences among 197 French farmers and structurally estimate these preferences. We use cumulative prospect theory and a multiple-prior model in order to model risk and ambiguity preferences, respectively. We find that farmers are risk, ambiguity, and loss averse, and that probability distortion differs in gains vs. losses, as well as in risk vs. ambiguity. These findings can have important implications for policy design.
\end{abstract}

Key words: Risk Preferences; Ambiguity Preferences; Uncertainty; Field Experiment; JEL: C93 (Field Experiments); D81 (Criteria for Decision-Making under Risk and Uncertainty); Q10 (Agriculture); 


\section{Introduction}

Risk is pervasive in farming activities, where producers face a variety of sources of uncertainty including those involving yields, input and output prices, agricultural policies, etc. Farmer attitudes towards uncertainty regarding yields and prices specifically, are an important topic for investigation for two main reasons. Uncertainty about yields can be exacerbated by environmental challenges that increase the likelihood of unusual climatic events and call into question the use of risk-reducing inputs such as pesticides. Uncertainty about prices is also an increasing concern among farmers due to the globalization of agricultural markets, which has caused prices in some parts of the world to be more sensitive to supply and demand shocks in other parts of the world, as well as due to the fact that governments have been reducing agricultural subsidies. In this changing context, it is very important for any economic analysis focusing on agriculture to correctly assess farmer behaviour in the face of these different sources of uncertainty.

While agricultural economists have long analysed uncertainty and attitudes towards uncertainty, the distinction between several related concepts, such as risk, uncertainty and ambiguity, has not always been clear-cut. For example, Moschini and Hennessy (2001, page 91) consider uncertainty reduces to risk: 'A modelling strategy that recurs in the applied literature is the distinction between uncertainty and risk attributed to Knight (1921). According to this view, risk arises when the stochastic elements of a decision problem can be characterized in terms of numerical objective probabilities, whereas uncertainty refers to decision settings with random outcomes that lack such objective probabilities. With the widespread acceptance of probabilities as subjective beliefs, Knight's distinction between risk and uncertainty is virtually meaningless and, like other authors [...], we will ignore it here.' The argument made by Moschini and Hennessy (2001) relies on the assumption that agents hold subjective beliefs with certainty. In many cases, however, agents do not have enough information to form any subjective belief distribution at all. Such situations are characterized as ambiguous, and in these situations, uncertainty does not reduce to risk (Harrison, 2011, pages 351-355). In this article we use the term risk to describe situations in which agents assign well-defined probabilities to outcomes, and ambiguity to describe situations in which agents do not ${ }^{1}$.

The development of studies in ambiguity in recent years is of particular interest in agricultural economics. Because most natural processes are ambiguous, technology adoption or extreme weather events may often involve not only risk, but ambiguity as well. In these cases, it becomes important for policymakers to consider both risk and ambiguity preferences. As pointed out by Chavas et al. (2010, p.370), one of the challenges for future research in agricultural economics is to better understand the role of risk and uncertainty in agriculture. Although ambiguity is of particular interest to agricultural and environmental economists, few papers in the agricultural and environmental field deal with ambiguity and those that do tend to be recent. To date, ambiguity has been studied in the context of four domains: agricultural technology adoption (Warnick et al., 2011; Barham et al., 2014), environmental management (Chevé and Congar, 2000; Hansen and Sargent, 2001; Roseta-Palma and Xepapadeas, 2004; Woodward and Shaw, 2008), food safety (Chambers and Melkonyan, 2013; Melkonyan and Schubert, 2009; Chambers and Melkonyan, 2007), and agricultural insurance (Frechette, 2000). We add to this literature by experimentally eliciting the ambiguity preferences of farmers in a developed country, namely France, using a carefully-designed experimental protocol.

The elicitation of risk preferences among farmers has mainly relied on revealed-

\footnotetext{
${ }^{1}$ Note that we focus on "lack of information regarding the true probability distribution, while the decision maker has full information regarding the set of outcomes" (Chakravarty and Roy, 2009, p.200)
} 
preference approaches. The advantage to this approach to deriving preferences is that they can be obtained from large samples that involve real economic decisions. The main drawback to the approach is that it relies on strong hypotheses, such as overweighting the role of diminishing marginal utility (Just and Pope, 2003), eliciting a distribution of parameters in a sample (versus individual parameters) and relying only on expected utility theory. Other researchers have elicited risk preferences using recent advances in experimental economics. There is indeed a recent and growing literature based on field experiments that use real payments to structurally derive risk preference parameters (Harrison and Rutström, 2008). Although they rely on smaller samples and provide less context than revealed-preference studies, experimental methods allow experimenters some degree of control over the decision maker's environment, enable the estimation of individual parameters using structural models, and provide alternatives to expected utility theory (Holt and Laury, 2002; Harrison and Rutström, 2008, 2009; Andersen et al., 2010; Tanaka et al., 2010). Few articles in this literature elicit preferences among farmer samples and those that do are mainly carried out in the context of developing countries (Binswanger, 1980; Humphrey and Verschoor, 2004; Harrison et al., 2010; Liu, 2013). Moreover, while risk preferences have been elicited in the literature to date (Bocquého et al., 2014; Hellerstein et al., 2013; Reynaud and Couture, 2012), very few papers elicit farmer preferences for ambiguity, perhaps because separately identifying preferences for risk and ambiguity is a challenging task. We aim to fill this gap by using recent advances in experimental economics to structurally elicit farmers' risk and ambiguity preferences.

To accomplish this, we design and conduct a field experiment ${ }^{2}$ and use these data to elicit the preference parameters of a utility model that distinguishes between risk and ambiguity. A total of 197 farmers participated in face-to-face interviews. We find that: (i) farmers are risk and ambiguity averse; (ii) farmers are loss averse; (iii) probability distortion differs significantly when facing gains vs. losses as well as when facing risky vs. ambiguous situations. These results highlight the importance of distinguishing not only between risk and ambiguity, but also between gains and losses when considering farmer preferences.

The paper is organized as follows. In section 2, we describe the structural models and empirically-derived specifications we use. In section 3, we describe the field experiment. Our results regarding the estimation of risk and ambiguity preferences are presented in Section 4. A discussion follows in Section 5 and Section 6 concludes.

\section{Structural models}

In this section we describe how we model preferences for risk and ambiguity ${ }^{3}$. Our goal is to separately identify risk preferences and ambiguity preferences, with a distinction in outcomes between gains and losses. In order to introduce our notation, we begin by presenting our modelling framework of risk (section 2.1). We use the cumulative prospect theory (CPT) developed by Tversky and Kahneman (1992), which allows for a distinction between gains and losses in risk preferences. In section 2.2, we introduce our model for ambiguity, namely a second order model developed by Klibanoff et al. (2005) that allows for a separation of risk and ambiguity. In section 2.3, we present the general model that

\footnotetext{
${ }^{2}$ In the typology of Harrison and List (2004), our experiment stands as an artefactual field experiment as it is 'the same as a conventional lab experiment but with a nonstandard subject pool.'

${ }^{3}$ We present structural models (in Section 2) before presenting the experimental protocol (in Section 3.2). Since there is close link between the two, interested readers may wish to take a first look at the experimental protocol in Appendix A.1
} 
allows for both a separation of risk and ambiguity as well as a distinction between gains and losses. Finally, in section 2.4, we present the empirical specifications derived from the structural models.

\subsection{Modelling decisions in a risky environment}

In this section, we present how we model decisions in a risky environment. While numerous alternatives to the expected utility theory (EUT) developed by von Neumann and Morgenstern (1947) can be found in the literature, we elect to use CPT to model risky decisions, as it has become one of the most commonly used in recent years. CPT extends the EUT in two directions: loss aversion ('losses loom larger than gains') and probability distortion. The utility function over wealth $x$ is written as in Equation (1).

$$
u(x)= \begin{cases}\left(x-x_{0}\right)^{\alpha^{+}} & \text {if } x \geq x_{0} \\ -\lambda \cdot\left[\left(-x+x_{0}\right)^{\alpha^{-}}\right] & \text {if } \quad x<x_{0}\end{cases}
$$

where $\alpha^{+}$is the concavity of the utility function for gains, $\alpha^{-}$is the concavity of the utility function for losses, $x_{0}$ is the reference point that defines the gain and loss domains, and $\lambda$ is a loss aversion parameter. Loss aversion as first proposed by Kahneman and Tversky (1979) is a model independent concept. Using a value function steeper for losses than for gains is one way, but not the only way, of capturing the concept of loss aversion (Schmidt and Zank, 2005).

Under CPT, probabilities are transformed by a probability weighting function, which we specify as:

$$
\Gamma(p)=\left\{\begin{array}{lll}
\frac{p^{\gamma^{+}}}{\left[p^{\gamma^{+}}+(1-p)^{\gamma^{+}}\right]^{1 / \gamma^{+}}} & \text {if } & x \geq x_{0} \\
\frac{p^{\gamma^{-}}}{\left[p^{\gamma^{-}}+(1-p)^{\gamma^{-}}\right]^{1 / \gamma^{-}}} & \text {if } & x<x_{0}
\end{array}\right.
$$

where $\gamma$ is a parameter describing the shape of the probability weighting function. Probability weighting functions differ in gains and losses. We estimate a parameter $\gamma^{+}$for gains and $\gamma^{-}$for losses. $\gamma<1$ implies an overweighting of small probabilities and an underweighting of high probabilities, resulting in an inverse S-shaped function, whereas $\gamma>1$ implies the opposite, resulting in an S-shaped function. Several probability weighting functions can be found in the literature. We choose the commonly-used function proposed by Tversky and Kahneman (1992) ${ }^{4}$. The specification defined by Equations (1) and (2) collapses to the EUT specification if $\lambda=1$ and $\gamma^{+}=\gamma^{-}=1$. This model constitutes the first step to modeling farmer risk preferences.

\subsection{Modelling decisions in an ambiguous environment}

Several theories model ambiguity. We use the multiple prior model, which belongs to the class of second-order models (SOMs). The intuition underlying a SOM is that decision under ambiguity corresponds to a decision over a compound lottery in which expectations about the ambiguous states of events must be resolved before expectations about the risky states can be formed. In these models, it is as if a second-order expectation is added by compounding ambiguity with the usual risky expectations. A SOM allows for a separation

\footnotetext{
${ }^{4}$ See Appendix A.2 for an alternative specification using Prelec (1998).
} 
between ambiguity, identified as a characteristic of the decision maker's subjective beliefs over the sampled lottery (represented by the distortion of the distribution probability), and the decision-maker's attitude towards this ambiguity, a characteristic of their tastes (represented by the utility function for ambiguity) (Klibanoff et al., 2005).

The SOM we use is based on Klibanoff et al. (2005), and more precisely on the discrete version of Nau (2006). The state space can be represented as a Cartesian product, $\mathcal{A} \times \mathcal{R}$, where $\mathcal{A}$ is the finite set of $J$ ambiguous elements and $\mathcal{R}$ is a set of $K$ risky elements. An act is a mapping from observable states of the world to quantities of a single consumption good and is denoted by a doubly-subscripted vector $\mathbf{x}=\left(x_{11}, x_{12}, \ldots, x_{J K}\right)$ where $x_{j k} \in \mathbb{R}$ are the payoffs in state $A_{j} R_{k}$. Under axioms $1,2,3^{*}$ and 4 detailed in Nau $(2006, \mathrm{p} 138,142-143)^{5}$, the preference relationship under risk and ambiguity holds if and only if the utility function is of the form found in Equation (3).

$$
E U(\mathbf{x})=\sum_{j=1}^{J}\left(\Phi_{j}\right) v\left(\sum_{k=1}^{K} \Gamma_{j k} u\left(x_{j k}\right)\right)
$$

where $E U$ is the overall expected utility, $\Phi=\left(\Phi_{1}, \ldots, \Phi_{J}\right)$ is a unique marginal probability distribution of ambiguity space $\mathcal{A}, \Gamma_{j}=\left(\Gamma_{j 1}, \ldots, \Gamma_{j K}\right)$ is a unique conditional probability distribution of risky space $\mathcal{R}$ given $\mathcal{A}_{j}, u$ is a strictly increasing state-independent first-order Bernoulli utility function unique up to positive affine transformations, and $v$ is a strictly increasing, state independent second-order Bernoulli utility function unique up to positive affine transformations given $u$. $\Phi$ and $\Gamma$ are subjective beliefs. The probability distribution $\Phi$ can be interpreted as the distribution of an individual's subjective beliefs about ambiguity. The function $v$ describes the ambiguity taste of the individual. Subjective beliefs about risky space $\Gamma$ are formed in the context of ambiguity. Under this model the decision maker therefore behaves as though he/she assigns probability $\Phi_{j} \Gamma_{j k}$ to the state $\mathcal{A}_{j} \mathcal{R}_{k}$, and bets on a risky event as though his/her utility function were $v(u()$.$) .$ The model follows the distinction made by Klibanoff et al. (2005) between ambiguity, described by the distribution $\Phi$, and attitude towards ambiguity, derived from the shape of the function $v$.

\subsection{Modelling decisions in a risky and ambiguous environment}

In Equation (4), we combine the two specifications of Equation (1) and Equation (3) in order to distinguish between gain and loss domains. As defined previously, $\mathbf{x}=$ $\left(x_{11}, x_{12}, \ldots, x_{J K}\right)$ is an act, $x_{j k}$ is an outcome, and $x_{0}$ is the reference point.

$$
E U(\mathbf{x})=\left\{\begin{array}{l}
\mathbf{I}\left(x_{j k} \geq x_{0}\right) \times \sum_{j=1}^{J} \Phi^{+}\left(q_{j}\right) \times v^{+}\left(\sum_{k=1}^{K} \Gamma^{+}\left(p_{j k}\right) \times u^{+}\left(x_{j k}-x_{0}\right)\right) \\
+\mathbf{I}\left(x_{j k}<x_{0}\right) \times \sum_{j=1}^{J} \Phi^{-}\left(q_{j}\right) \times v^{-}\left(\sum_{k=1}^{K} \Gamma^{-}\left(p_{j k}\right) \times(-\lambda) u^{-}\left(-x_{j k}+x_{0}\right)\right)
\end{array}\right.
$$

We define the parameters as follows. $u^{+}$and $u^{-}$are the utility functions for risk in the gain domain and the loss domain, respectively. $v^{+}$and $v^{-}($.$) are the utility functions$ for ambiguity in the gain and loss domains, respectively. $\Gamma^{+}$and $\Gamma^{-}$are the probability weighting functions in the gain and loss domains, respectively. Finally, $\Phi^{+}$and $\Phi^{-}$are the probability weighting functions on the distribution of probabilities in the gain and loss

\footnotetext{
${ }^{5}$ We do not present the set of assumptions and axioms here due to space constraints. Interested readers may wish to refer to Klibanoff et al. (2005) and Nau (2006) for more details.
} 
domains, respectively ${ }^{6} . \lambda$ is the loss aversion coefficient: $\mathbf{I}\left(x_{j k} \geq x_{0}\right)$ and $\mathbf{I}\left(x_{j k}<x_{0}\right)$ are indicator functions that take the value 1 if $x_{j k} \geq x_{0}$ or $x_{j k}<x_{0}$, and take the value 0 otherwise.

Utility functions and probability weighting functions are defined as in Equation (6).

$$
\begin{aligned}
& u^{+}(x)=x^{\alpha^{+}} \quad u^{-}(x)=x^{\alpha^{-}} \\
& v^{+}(x)=x^{\rho^{+}} \quad v^{-}(x)=x^{\rho^{-}} \\
& \Gamma^{+}(p)=\frac{p^{\gamma^{+}}}{\left(p^{\gamma^{+}}+(1-p)^{\gamma^{+}}\right)^{1 / \gamma^{+}}} \quad \Gamma^{-}(p)=\frac{p^{\gamma^{-}}}{\left(p^{\left.\gamma^{-}+(1-p)^{\gamma^{-}}\right)^{1 / \gamma^{-}}}\right.} \\
& \Phi^{+}(q)=\frac{q^{\phi^{+}}}{\left(q^{\phi^{+}}+(1-q)^{\phi^{+}}\right)^{1 / \phi^{+}}} \quad \Phi^{-}(q)=\frac{q^{\phi^{-}}}{\left(q^{\left.\phi^{-}+(1-q)^{\phi^{-}}\right)^{1 / \phi^{-}}}\right.}
\end{aligned}
$$

The aim of this article is to estimate the parameters associated with these functions using field experiments involving binary lottery choices. The more parameters to be estimated, the greater the number of lottery choice series needed to estimate these parameters. Given the constraints associated with survey implementation (time constraints and subject fatigue), we sought to use the fewest number of series as possible. We employ the design developed by Chakravarty and Roy (2009), which allows the model to be identified with a fairly reasonable number of lottery choices series. Chakravarty and Roy (2009) uses a design in which ambiguity preferences are estimated using lotteries that involve ambiguity but not risk, which reduces the number of series of lottery choices needed in our study. Subjects are faced either with lottery choices involving risk and no ambiguity, or with lottery choices involving ambiguity and no risk. We do not need to estimate Equation (6). Instead, we estimate two equations: Equation (7) for risky situations without ambiguity and Equation (8) for ambiguous situations without risk.

$$
\begin{gathered}
E U(\mathbf{x})=\left\{\begin{array}{l}
\mathbf{I}\left(x_{j k} \geq x_{0}\right) \times\left(\sum_{k=1}^{K} \Gamma^{+}\left(p_{j k}\right) \times u^{+}\left(x_{j k}-x_{0}\right)\right) \\
+\mathbf{I}\left(x_{j k}<x_{0}\right) \times\left(\sum_{k=1}^{K} \Gamma^{-}\left(p_{j k}\right) \times(-\lambda) u^{-}\left(-x_{j k}+x_{0}\right)\right)
\end{array}\right. \\
E U(\mathbf{x})=\left\{\begin{array}{l}
\mathbf{I}\left(x_{j k} \geq x_{0}\right) \times \sum_{j=1}^{J} \Phi^{+}\left(q_{j}\right) \times v^{+}\left(x_{j k}-x_{0}\right) \\
+\mathbf{I}\left(x_{j k}<x_{0}\right) \times \sum_{j=1}^{J} \Phi^{-}\left(q_{j}\right) \times(-\lambda) v^{-}\left(-x_{j k}+x_{0}\right)
\end{array}\right.
\end{gathered}
$$

The model collapses to CPT for a compound lottery under risk if and only if the utility function for risk and the utility function for ambiguity are the same, and the distortion for probability and distribution are also the same: $v^{+}=u^{+}, v^{-}=u^{-}, \Gamma^{+}=\Phi^{+}$and $\Gamma^{-}=\Phi^{-}$. The model can collapse further to EUT if $v^{+}=v^{-}=\mathbb{I}$, the identity function (i.e. $\mathbb{I}(x)=x \forall x), \lambda=1$ and $\gamma^{+}=\gamma^{-}=1$. CPT and EUT are therefore restrictions of this model ${ }^{7}$. Our model thus allows for a direct testing of whether these restrictions hold. As noted by Barberis (2013), it is difficult to know how to precisely define what constitutes a gain or loss, which leads us to set the reference point $x_{0}=0$.

\footnotetext{
${ }^{6}$ In the literature, constraints are usually set on $\phi^{+}$and $\phi^{-}$. In Chakravarty and Roy (2009), the two parameters are assumed to be equal; $\phi^{+}=\phi^{-}=1 / 2$. Andersen et al. (2009) discuss the identification issues raised by this specification and the associated experimental design for the case where ambiguity aversion $\rho$ and the distribution distortion $\phi^{+}=\phi^{-}$cannot be estimated independently. We did not impose any constraints on $\phi^{+}$and $\phi^{-}$in order to better disentangle ambiguity preferences from distribution distortion.

7 Note that we use the same parameter to capture loss aversion in both the risk and the ambiguity domain. We make this assumption in order to be able to compare risk attitudes and ambiguity attitudes. We find support for this assumption in Abdellaoui et al. (2016), who find no significant difference between loss aversion in risk and loss aversion in ambiguity.
} 


\subsection{Empirical specification}

The experimental protocol that we design allows us to minimize the number of binary choice lotteries presented to subjects in the experiment. As mentioned in section 2.3, the series of lottery choices were designed so that risky choices involved no ambiguity and ambiguous choices involved no risk. The empirical specification is similar for these two situations. First let us consider risky lotteries without ambiguity (for simplicity of exposition, we drop the superscript indicating the gain or loss domain).

Farmers faced a series of lottery choices. In each lottery choice situation $j$, farmers chose between two risky lotteries A and B: $\left\{\left(p_{j}^{A}, x_{j H}^{A}, x_{j L}^{A}\right) ;\left(p_{j}^{B}, x_{j H}^{B}, x_{j L}^{B}\right)\right\}$. Lottery A offers a high outcome $x_{j H}^{A}$ with probability $p_{j}^{A}$ and a low outcome $x_{j L}^{A}$ with probability $1-p_{j}^{A}$. Lottery B offers a high outcome $x_{j H}^{B}$ with probability $p_{j}^{B}$ and a low outcome $x_{j L}^{B}$ with probability $1-p_{j}^{B}$.

For lottery choice $j$, an individual facing lottery $k \in\{A, B\}$ obtains the utility described in Equation (9).

$$
U^{k}=\Gamma\left(p_{j}^{k}\right) \cdot u\left(x_{j H}^{k}\right)+\Gamma\left(1-p_{j}^{k}\right) \cdot u\left(x_{j L}^{k}\right)
$$

where $\Gamma$ is the probability weighting function and $u$ is the value function.

We consider a Fechner stochastic model ${ }^{8}$ and assume that subjects make errors in evaluating the expected utility of lotteries (Loomes et al., 2002). The difference in utilities $\Delta U=U^{B}-U^{A}$ between the two lotteries provides the rule for an individual choosing lottery B. We model the decision as a discrete choice model (Harrison and Rutström, 2008).

Subject $i$ chooses lottery B if $U^{B}-U^{A}+\epsilon>0$ and lottery A otherwise with $\epsilon$ as a random component normally distributed with zero mean and variance $\sigma^{2}$. We consider a latent variable $d^{*}=\Delta U+\varepsilon$ that describes the decision to choose lottery $\mathrm{B}$. We do not observe $d^{*}$ but only the choices $d$ that individuals make, so that the rule is as in Equation (10).

$$
\left\{\begin{array}{l}
d=1 \text { if } d^{*}>0 \\
d=0 \text { if } d^{*} \leq 0
\end{array}\right.
$$

Let $Z=\frac{\epsilon}{\sigma}$. By definition, $\mathrm{Z}$ is normally distributed with a mean of zero and a variance of one. The probability of choosing lottery B therefore writes as in Equation (11) with $F(\cdot)$ as the standard normal distribution function. The probability of choosing lottery A writes as in Equation (12).

$$
\begin{gathered}
\operatorname{Prob}(B)=\operatorname{Prob}\left(d^{*}+\epsilon>0\right)=\operatorname{Prob}\left(Z>-\frac{d^{*}}{\sigma}\right)=F\left(d^{*} / \sigma\right) \\
\operatorname{Prob}(A)=\operatorname{Prob}\left(-d^{*}+\epsilon>0\right)=1-F\left(d^{*} / \sigma\right)
\end{gathered}
$$

We estimate five parameters with maximum likelihood: risk aversion $\alpha$, loss aversion $\lambda$, probability distortion in gains $\gamma^{+}$, probability distortion in losses $\gamma^{-}$and the standard deviation of the Fechner error term $\sigma$.

\footnotetext{
${ }^{8} \mathrm{We}$ expect the specification of an error term to modify the estimated parameters (Loomes et al., 2002). We carried out maximum likelihood estimations without any error specification. As expected, results indicate that our model is not robust to the introduction of a stochastic error specification. Specifically, the model without error over-estimates risk aversion and ambiguity aversion parameters.
} 
The log likelihood function writes as in Equation (13) where $\mathbf{I}(\cdot)$ is the indicator function, $d_{i j}=1$ when lottery $\mathrm{B}$ is chosen by individual $i$ in lottery choice $j$ and $d_{i j}=0$ when lottery $\mathrm{A}$ is chosen. The value of $\Delta U$ is the difference in utilities between the two lotteries.

$\ln \left(L\left(\alpha, \lambda, \gamma^{+}, \gamma^{-}, \sigma\right)\right)=\sum_{i}\left\{\left[\ln \left(F\left(d^{*} / \sigma\right)\right)\right] . \mathbf{I}\left(d_{i j}=1\right)+\left[\ln \left(1-F\left(d^{*} / \sigma\right)\right)\right] . \mathbf{I}\left(d_{i j}=0\right)\right\}$

A similar empirical strategy is used for ambiguous lotteries without risk. The underlying functional form parameters $\left(\rho, \phi^{+}, \phi^{-}, \sigma\right)$ are estimated using maximum likelihood.

Recall that the specifications for risky situations without ambiguity and for ambiguous situations without risk are presented in Equation (7) and Equation (8), respectively.

\section{The field experiment}

The field experiment took place in the winter of 2011 in the region of ChampagneArdenne, in north-eastern France. This area is one of the most intensively farmed regions in the country, where $61.4 \%$ of land is dedicated to agriculture.

We randomly chose farmers from a list of members of a cooperative. For homogeneity reasons, we excluded from the list the few farmers who farmed non-chalk soils. The cooperative provided us with a list of 1,658 farmers. We send a letter to 851 farmers randomly chosen in the list to inform them of an upcoming phone call. Among the 851 farmers, some could not be contacted by phone (wrong phone number), others were not available and 198 chose to participate. The enumerators did not keep track of the difference between those who had a wrong phone number and those who refused to participate, so that the only figure we can give for participation rate is the following one: 198 out of 1,658 farmers agreed to participate in the survey. Almost one fourth $(23 \%)$ of the contacted farmers agreed to participate ${ }^{9}$. Farmers participated in face-to-face interviews at the cooperative located nearest to their farmstead. Each interview lasted about one and a half hours. The questionnaire was composed of the series of lottery choices followed by a survey to collect data on the characteristics of the farmer and the farm. First, we describe the sample and second, we describe the protocol used in the field experiment.

\subsection{Sample description}

We collected questionnaires from 197 farmers (one farmer out of the 198 participants left the experiment for personal reasons). Most of them (128 or 65\%) produced cereals, oilseeds, protein crops (COP) and sugar beets, while 33 (17\%) also produced field vegetables (potatoes, onions, etc.), 34 (17\%) were engaged in mixed crop and livestock farming and $2(1 \%)$ specialized in vineyards. Table 1 reports the average values for several important structural variables describing our sample. In order to assess the representativeness of our sample, the table also reports the corresponding averages for all farms both at the Champagne-Ardenne region level and for France as a whole, as well as for comparable

\footnotetext{
${ }^{9}$ This participation rate is lower than in other studies. Using the same calculation as ours, that is including farmers with wrong profession or contact information, Bocquého et al. (2014) find a $46 \%$ participation rate. Trujillo-Barrera et al. (2016) find a $40 \%$ participation rate. Since we have no information on non participants, it is difficult to understand why our participation rate is lower. Note that incentive levels do not explain the participation rate since, in the letter, participants were not informed of the incentives but only of a 20 euro show-up fee designed to cover expenses for travel and time.
} 
farms both in terms of productive specialization (COP) and economic size ('large' farms, i.e., those whose Standard Output (SO) is higher than or equal to 100,000 Euros). Column (a) of Table 1 reveals that farmers in our sample were almost exclusively men (97\%). They were on average 49 years old and exhibited a high level of education, as $65 \%$ of them possess a 'baccalauréat', the French secondary school diploma. They operated on average almost 160 hectares each, but farm size varied greatly, ranging from 1.28 to 790 hectares (not reported), with a standard deviation of almost 98 hectares. Most farms in our sample were run as a company (70\%) with less than one-fourth run as a partnership (23\%) and very few run as individual holdings (7\%).

With these characteristics, our sample can be considered as representative of 'large' farms, especially those specialized in COP in the Champagne-Ardenne region (column (c) of Table 1), that is, farms that are a priori comparable when considering the composition of our sample as described above. Indeed, values between this group and our sample appear to be very close in terms of the gender, average acreage, and especially legal status. The only major difference between our sample and the general population is that farmers in our sample appear to be twice as educated as the average farmer. Although the breakdown of legal statuses in our sample is somewhat less similar to that of the population of farmers, our sample is similar to 'large' COP farms in France as a whole in this respect (column (g) of Table 1). However, our sample cannot be regarded as representative of other types of farms. In particular, the sample farms depart from average farm characteristics in Champagne-Ardenne and in France more generally (columns (d) and (h) of Table 1), and even from average COP farms of all commercial sizes (column (e) and (i) of Table 1) in several ways. Namely, our sample is characterized by a higher proportion of men, a higher average level of education among farmers, a larger average farm size, and an overrepresentation of companies relative to individual farms. Age does not appear to be a discriminating criteria since it is fairly homogeneous, with an average of about 50 across all types of farms considered in Table 1. 


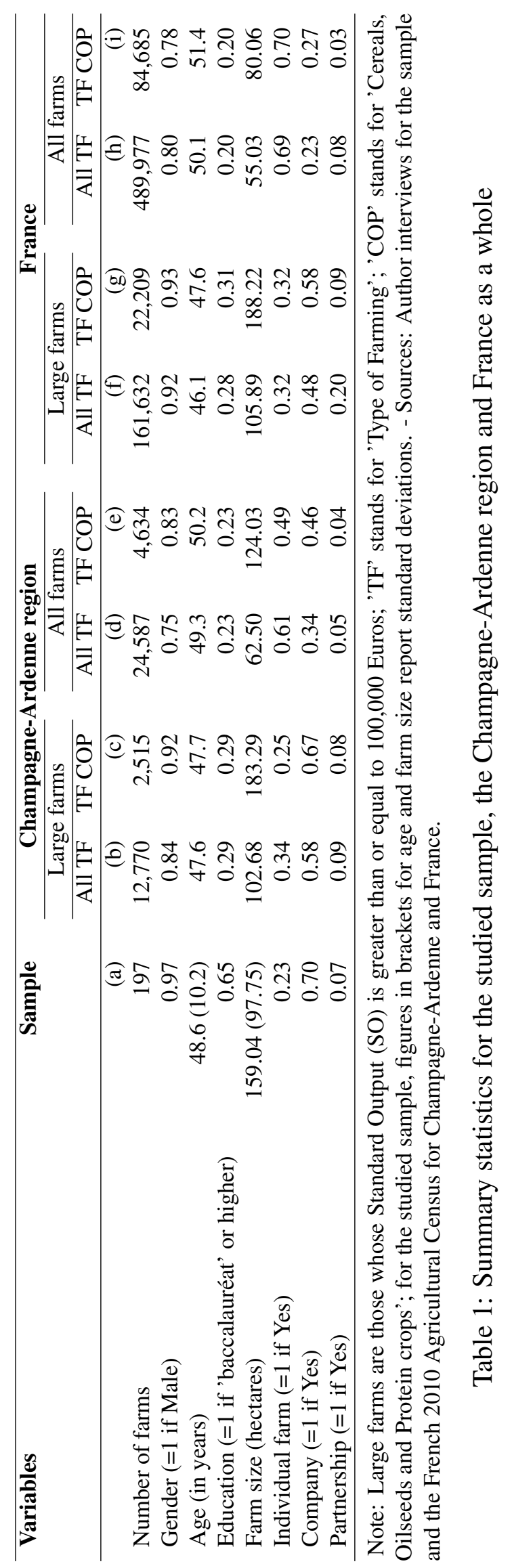




\subsection{The lottery choices}

For the section containing lottery choices, we used a multiple price list procedure according to which farmers made a series of choices between two lotteries with varying probabilities and outcomes in the gain and loss domains. The choices are presented following the format of Table 2 for risk and Table 3 for ambiguity. The complete questionnaire is reported in Appendix A.1. Before facing the first risky lottery and the first ambiguous lottery, an example was presented to farmers describing how the lottery choices worked and what was expected of them. Following the example, farmers were asked to answer some simple questions in order to assess their understanding of the lottery tasks.

\subsubsection{Lottery choice design}

Table 2 presents an example of a series of ambiguity-free but risky lottery choices. In each row, farmers had to choose between lottery A (two probabilities and two outcomes) and lottery B (two probabilities and two outcomes). We did not allow for indifference nor impose a single switching point. Multiple switching points occur when a subject switches more than once from one lottery to the other (in our design, from urn A to urn B) in the same series. We chose to let our subjects behave as freely as possible, as we consider this to be most similar to real life decisions, and so did not impose any restrictions on switching. Ex-post, we will compare parameters estimated using the whole sample vs parameters estimated excluding subjects that made at least two switchings in at least one of the 9 series.

Table 2 provides an example (See Table A.1 in Appendix A.1). The four probabilities are invariant. Three outcomes out of four are invariant. The fourth outcome increases from row to row.

\begin{tabular}{|c|c|c|c|c|c|}
\hline \multicolumn{2}{|c|}{ Urn A } & \multicolumn{2}{|c|}{ Urn B } & \multirow{2}{*}{\multicolumn{2}{|c|}{ Choice }} \\
\hline $3 / 10$ & $7 / 10$ & $1 / 10$ & $9 / 10$ & & \\
\hline $200 €$ & $100 €$ & $270 €$ & $75 €$ & A & B \\
\hline $200 €$ & $100 €$ & $280 €$ & $75 €$ & $\mathrm{~A}$ & $\mathrm{~B}$ \\
\hline $200 €$ & $100 €$ & $350 €$ & $75 €$ & $\mathrm{~A}$ & $\mathrm{~B}$ \\
\hline $200 €$ & $100 €$ & $390 €$ & $75 €$ & A & B \\
\hline $200 €$ & $100 €$ & $430 €$ & $75 €$ & $\mathrm{~A}$ & B \\
\hline $200 €$ & $100 €$ & $450 €$ & $75 €$ & $\mathrm{~A}$ & B \\
\hline $200 €$ & $100 €$ & $480 €$ & $75 €$ & A & B \\
\hline $200 €$ & $100 €$ & $520 €$ & $75 €$ & $\mathrm{~A}$ & B \\
\hline $200 €$ & $100 €$ & $600 €$ & $75 €$ & $\mathrm{~A}$ & B \\
\hline $200 €$ & $100 €$ & $700 €$ & $75 €$ & $\mathrm{~A}$ & B \\
\hline $200 €$ & $100 €$ & $900 €$ & $75 €$ & $\mathrm{~A}$ & B \\
\hline $200 €$ & $100 €$ & $1,200 €$ & $75 €$ & A & B \\
\hline $200 €$ & $100 €$ & $2,200 €$ & $75 €$ & $\mathrm{~A}$ & B \\
\hline $200 €$ & $100 €$ & $3,000 €$ & $75 €$ & $\mathrm{~A}$ & B \\
\hline
\end{tabular}

Table 2: Series RG1 (risk in gain domain)

The elicitation process is simple. Urn A is fixed, with low risk and low reward, whereas urn B is more risky, starting with a low reward in the first row and finishing with a high reward in the last row. The idea behind this elicitation strategy is to reveal the amount needed for a farmer to switch from urn A to urn B. The row at which farmers switch from urn A to urn B is referred to as the switching point, which provides information about a farmer's preferences. For example, consider a risk neutral subject without distortion of probabilities. This subject only takes into consideration the expected value of the lottery when making his/her choices. In the case of Table 2, the expected value of urn $\mathrm{A}$ is greater than the expected value of urn B for rows 1 to 9 , and the reverse is true for rows 10 to 14. The switching point for this particular agent would therefore occur at row 10. The switching point for such a risk-neutral subject is called the neutral switching 
point. Farmers who switched before the neutral switching point are considered to be risk seeking whereas farmers who switched after the neutral switching point are considered to be risk averse.

We note that a framing effect may occur in such a multiple price list design if subjects tend to preferentially choose to switch at the middle row. Indeed, evidence shows that subjects can be influenced by the bounds of the array (i.e. the first and the last row) and may consider these bounds as some sort of reference point that they should not go beyond, which may thus lead them to prefer the middle row (Harrison et al., 2005b). In order to control for such framing effects, we designed the nine series so that the row position of the neutral switching point was not systematically the middle row, but rather varied across the series.

Table 3 provides an example (See Table A.5 in Appendix A.1). Urn A is a risky lottery without ambiguity, whereas urn B is an ambiguous lottery without risk. No objective probabilities were given to the subject concerning urn $\mathrm{B}$; only the distribution was known. At the end of the experiment, if urn B is played for earnings, subjects know with certainty the outcome of urn B once the ambiguity on the distribution is resolved, as no additional randomization or risk is present for this lottery. For this reason, urn B is not risky. At the beginning of an ambiguous task farmers were asked to choose the colour they wished to bet on. Their gains were determined according to the match between the chosen colour and the colour eventually drawn. In this way we controlled for subjects' colour preferences, since they could always choose their favourite colour without distorting their preferences between urn A and urn B. As in the risk domain, we computed the neutral switching point for ambiguity so that the point at which farmers switched from urn A to urn B revealed their ambiguity preferences.

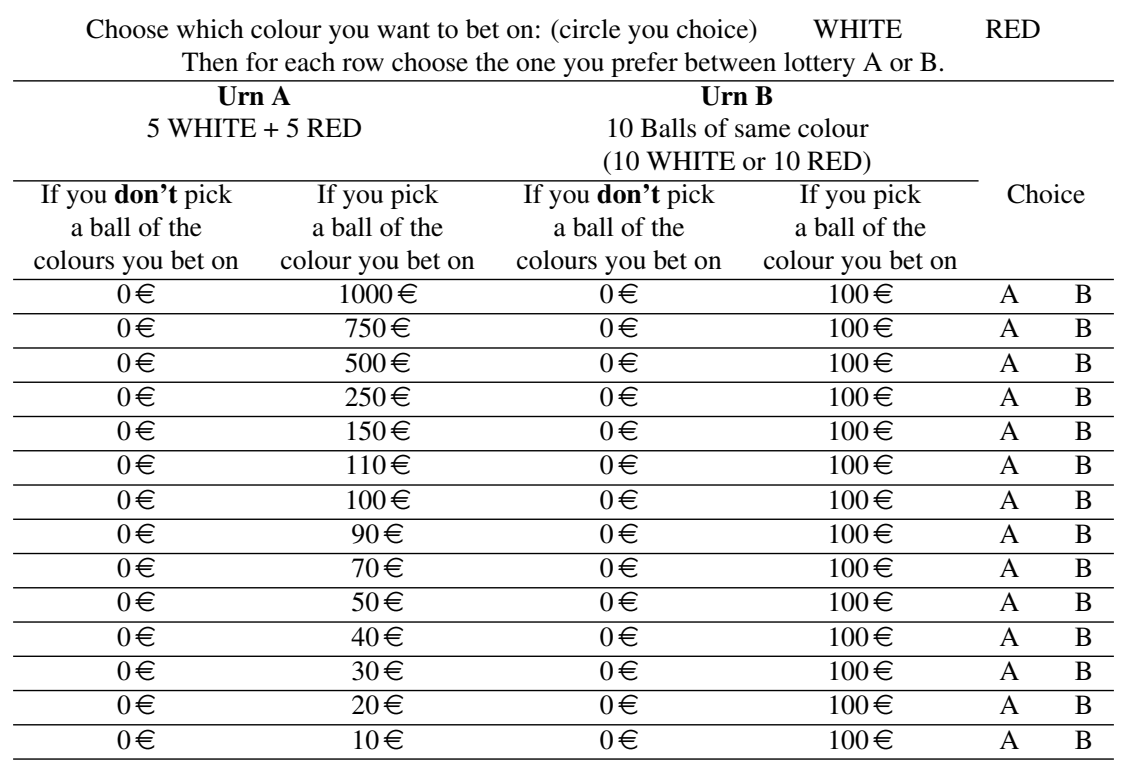

Table 3: Series AG1 (ambiguity in gain domain)

Nine is the minimum number of series needed to elicit the nine parameters we intend to estimate: two parameters for risk aversion in the gain $\left(\alpha^{+}\right)$and loss $\left(\alpha^{-}\right)$domains; two parameters for probability distortion in the gain $\left(\gamma^{+}\right)$and loss $\left(\gamma^{-}\right)$domains; one parameter for loss aversion $(\lambda)$; two parameters for ambiguity aversion in the gain $\left(\rho^{+}\right)$ and loss $\left(\rho^{-}\right)$domains; and two parameters for probability distortion of the distributions in the gain $\left(\phi^{+}\right)$and loss $\left(\phi^{-}\right)$domains. 
Loss aversion is characterized by the fact that "losses loom larger than gains" (Tversky and Kahneman, 1992). The 3-parameter value function in Equation (1) differs from the models usually estimated in the literature. Indeed, most studies involving a structural estimation of risk parameters (Bocquého et al., 2014; Tanaka et al., 2010) assume that $\alpha^{+}=\alpha^{-}$. In order to facilitate a comparison of our results with the existing literature, we make the same assumption that $\alpha=\alpha^{+}=\alpha^{-}$, as well as the assumption that $\rho=\rho^{+}=\rho^{-}$(see Section 4.1). Appendix A.3 presents the results when these equality assumptions are relaxed.

We therefore use the following nine series (the questionnaire is available in Appendix A.1):

- Series RG1 and RG2: These series involve 14 choices each. Each series is in the gain domain under risk, with the same sets of probabilities (0.9/0.1 for urn A and $0.7 / 0.3$ for urn B) but different outcomes. These two series enable us to estimate two parameters: a curvature $(\alpha)$ and a probability distortion of risk in the gain domain $\left(\gamma^{+}\right)$.

- Series RGL: This series involves 7 choices in both the gain and the loss domain under risk. Once we obtain the curvature of the utility function from series RG1 and RG2, series RGL enables us to estimate a parameter for loss aversion $(\lambda)$.

- Series RL1 and RL2: These series involve 14 choices each in the loss domain under risk, with the same probabilities $(0.9 / 0.1$ for urn A and $0.7 / 0.3$ for urn B) but different outcomes. These two additional series enable us to estimate an additional parameter: a probability distortion of risk in the loss domain $\left(\gamma^{-}\right)$.

- Series AG1 and AG2: These series involve 14 choices each in the gain domain under ambiguity, with the same distributions of probabilities. Series AG1 involves urns with 2 colours, while series AG2 involves urns with 3 colours. Once we determine the curvature of the utility function under risk from the previous series, these two series enable us to estimate two additional parameters: a curvature $(\rho)$ and a distribution distortion in the gain domain $\left(\phi^{+}\right)$under ambiguity.

- Series AL1 and AL2: These two series mirror series AG1 and AG2 but in the loss domain. Once we obtain the curvature of the utility function under risk from previous series, these two series enable us to estimate another parameter: a distribution distortion in the loss domain under ambiguity $\left(\phi^{-}\right)$.

With these series, we have a total of 119 lottery choices for each farmer. Series RG1, RG2, RGL, RL1 and RL2 constitute a variation of the protocol used by Tanaka et al. (2010) and series AG1, AG2, AL1 and AL2 constitute a variation of the protocol used by Chakravarty and Roy (2009). We control for possible order effects in the presentation by randomizing the order in which the nine series are presented.

\subsubsection{Incentives}

Following the practice in experimental economics work, our experiment was incentivized. All participants received a real payment that consisted of two parts, a fixed show-up fee (20€) to cover the expenses associated with attending the experiment, and a variable amount of money determined by their lottery earnings.

Subjects were informed before the experiment that one of the 119 lotteries would be randomly selected and that the urn that was chosen in the selected lottery (A or B) 
would actually be played for payment at the end of the interview. In order to ensure homogenous incentives, all participants in our experiment played for these earnings. Due to budget constraints, some experimentalists randomly select a subset of subjects who actually play the lottery for payment. These researchers argue that subjects are still incentivized in the experiment since each subject faces the same probability of winning. However, this rationale holds only if subjects have homogeneous risk preferences. If this is not the case, then incentives effectively vary across subjects, which results in a loss of control over participant incentives. We choose to have all participants actually play the lottery for payment because we wished to avoid introducing an additional layer of risk to our design and sacrificing control over incentives. Accordingly, each farmer received a show-up fee and his/her gains/losses based on his/her stated choices. This ensured a more homogeneous incentive structure for all farmers.

In the presented series, farmers were offered relatively high payoffs. However, budgetary constraints required us to divide the payoff of each lottery by 50 in order to ensure the financial feasibility of the experiment ${ }^{10}$. Overall, the highest amount that a subject could earn ${ }^{11}$ was $60 €$ while the lowest was a loss of $20 €$. On average, farmers actually received $21.21 €($ SD $6.69 €)$ including the $20 €$ show-up fee.

\section{Empirical results}

In this section ${ }^{12}$, we present the elicited preferences of our sample of 197 farmers. We specify a utility function under two different theoretical frameworks. In section 4.1, we consider CPT, thus adding the possibility for both loss aversion and probability distortion as compared to EUT. Then, in section 4.2, we consider a SOM that also encompasses ambiguity. Presenting our results in this way, from the simpler to the more generalized model, facilitates comparison with existing studies and highlights the marginal improvements in understanding preferences. The main results of this section are reported in Table 4, which presents the maximum likelihood (ML) estimation, with individual clustering, of risk, ambiguity and probability weighting parameters under the two chosen theoretical models, namely CPT and SOM. Each of these theoretical frameworks is analysed in detail in the following sub-sections ${ }^{13}$.

In each case, estimations only involved 23, 442 observations rather than $119 \times 197=$ 23,443 , due to one missing observation. We also estimate the Fechner error standard deviation, $\sigma$, in all specifications, which was significantly different from zero in all models, suggesting that preferences are quite heterogeneous in our sample.

\footnotetext{
${ }^{10} \mathrm{We}$ used this protocol for two reasons. First, budget constraints prevented us from offering such high payments. This could be a serious issue since, as argued by Rabin (2000), low stakes can result in riskneutral behaviour. In order to counteract this effect we multiplied the amounts displayed in the experiment by a scale factor. Second, we followed a protocol similar to those used in other experimental papers. Several scaling factors that have already been used in the literature are 2\% in Bocquého et al. (2014), 10\% in Abdellaoui et al. (2008) and 1/600 in Galarza (2009). Scaling the displayed earning amounts, however, does make it difficult to know with certainty whether subjects made their decisions based on the high amounts that were displayed or the lower amounts that were actually to be paid at the end of the experiment.

${ }^{11}$ Due to financial constraints, we recognize that the incentives in our experiment are relatively weak as compared to farmers' income. We note that our protocol may also suffer from house money effects (Cárdenas et al., 2014).

${ }^{12}$ We provide summary information on subjects' lottery choices in Appendix A.4.

${ }^{13}$ We also have considered a simple expected utility model as a first step. We report the results in Appendix A.5.
} 


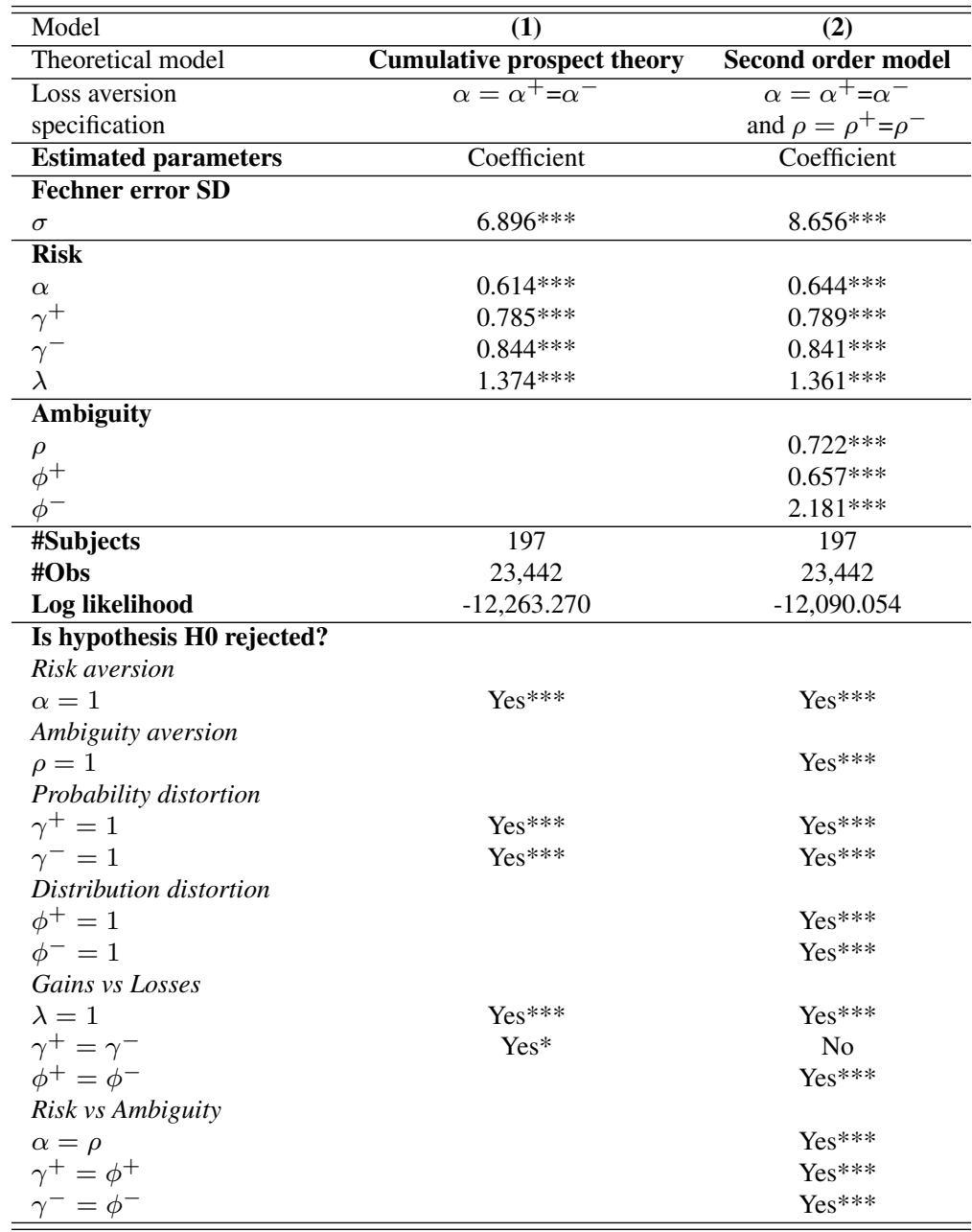

*** significant at $1 \%$ level; ** significant at $5 \%$ level; * significant at $10 \%$ level.

Table 4: ML estimations of farmers' risk and ambiguity preferences (197 farmers)

\subsection{Risk preferences under Cumulative Prospect Theory}

In model (1) in Table 4, we assume ${ }^{14}$ that $\alpha=\alpha^{+}=\alpha^{-}$so that loss aversion is captured through parameter $\lambda$. Results indicate that farmers are risk averse $(\alpha=0.614)$ and loss averse $(\lambda=1.374)$, and post-estimation tests find that $\lambda>1$. Similarly, we find that farmers in our sample exhibit an inverse S-shaped function for probability distortion of gains and losses. This means that farmers in the sample tend to overweight small probabilities and underweight high probabilities in both domains. Further, we find significantly less probability distortion in losses than in gains, since $\gamma^{-}$is significantly greater than $\gamma^{+}$. Recall that our CPT specification collapses to EUT if $\lambda=1$ and $\gamma^{+}=\gamma^{-}=1$. Results for model (1) lead to the rejection of EUT. We therefore conclude that CPT offers a better description of farmers' preferences than EUT.

We are tempted to compare our results with previous findings in the litterature. However, one needs to be careful since we must ensure consistent specification, similar experimental design and same estimation method before undertaking comparison of estimates.

Only one study, Tversky and Kahneman (1992), uses the same specification but with

\footnotetext{
${ }^{14}$ We can also specify a model where $\lambda=1$ and where $\alpha^{+}$and $\alpha^{-}$differ and $\rho^{+}$and $\rho^{-}$differ in order to test for preference reversal. Appendix A.3 presents the results when the assumption of equality of parameters is relaxed. Assuming $\lambda=1$, we find evidence of preference reversal: $\alpha^{+} \neq \alpha^{-}$and $\rho^{+} \neq \rho^{-}$ (1\% significant).
} 
a sample of students. They find $\alpha^{+}=\alpha^{-}=0.88, \lambda \approx 2.25, \gamma^{+} \approx 0.61$ and $\gamma^{-} \approx 0.69$. These results are consistent with our findings. The magnitude of parameter estimates differ notably because samples differ in characteritics such as gender or income known to determine risk aversion (although income should not play a role because we used a CRRA value function). It could also be that individuals self-select into activities according to their risk preferences (high education, farming). We would need a structural model to derive sound conclusions on why estimates differ between samples.

Consider now studies using a Prelec function instead of a Tversky-Kahneman function for probability distortion. The study that uses a sample close to ours is Bocquého et al. (2014) who estimated a risk aversion parameter of around 0.28 for French farmers. As shown in Appendix A.2, we report a 0.634 risk parameter with a Prelec function. Our sample is less risk averse. The difference may lie in differences in farm or farmer characteritics. Our sample is more educated and is less in individual ownership status. These differences and others may explain the differing risk attitudes. Here also, unless we use a structural model, it is difficult to explain the differences. Two other studies use a Prelec function but with samples very different from ours: Liebenehm and Waibel (2014) who find a value of 0.112 for cattle farmers in Burkina Faso and Nguyen (2011) who find a value of 1.012 for villagers in Vietnam. Harrison et al. (2010) use a Tversky-Kahneman function for probability distortion but a value function different from ours on a sample of villagers from Uganda, India and Ethiopia. They find a risk aversion parameter of 0.464.

Finally, consider three studies using a Prelec function but without ML estimation. Our sample has a risk aversion parameter similar to that of: Tanaka et al. (2010) who find a value of 0.60 and Nguyen and Leung (2010) who find a value of 0.62 both for villagers in Vietnam; Liu (2013) who find a value of 0.52 for villagers in China ${ }^{15}$.

As for probability distortion, our farmers exhibit a probability distortion similar to Bocquého et al. (2014) who find a value of 0.66 with a Prelec function. It is also similar to Liu (2013) who find a value of around 0.69, Nguyen and Leung (2010) who find a value of 0.75 , Tanaka et al. (2010) who find a value of 0.74 all with a Prelec function and no ML estimation. Note that these studies do not distinguish between domains (gains vs. losses). Harrison et al. (2010) find an S-shaped distortion function (estimated parameter is 1.384) and stand in contrast with our results and the results of the litterature.

As for loss aversion, our estimated $\lambda$ parameter with a Prelec function $\lambda=1.380$ is lower than the value of 2.28 found by Bocquého et al. (2014) on a similar sample. On a different sample, Liebenehm and Waibel (2014) find a similar value $\lambda=1.351$. With a Prelec function and without ML estimation, Tanaka et al. (2010), Liu (2013) and Nguyen and Leung (2010) find higher values 2.63, 3.47, 2.05 respectively.

In real world situations, however, farmers generally possess little information about event probabilities. In section 4.2, we therefore further extend our modelling framework to include the estimation of ambiguity preferences in order to improve our understanding of farmer preferences.

\subsection{Risk and ambiguity preferences under a Second Order model}

In this section, we use the model described in Equations (7) and (8). In model (2) in Table 4, we assume that $\alpha=\alpha^{+}=\alpha^{-}$and $\rho=\rho^{+}=\rho^{-}$. Estimation results are presented in the second column of Table 4.

\footnotetext{
${ }^{15}$ Computed using our specification for the value function; Liu (2013) use a value function that slightly differs from ours.
} 
First we consider risk attitudes. We find that farmers are risk averse in the gain domain and risk seeking in the loss domain, with $\alpha<1$, and we also find evidence of loss aversion. Farmers display an inverse $S$-shaped probability distortion in gains $\left(\gamma^{+}<1\right)$ and losses $\left(\gamma^{-}<1\right)$. However, we find that the distortions in the gain and loss domains are not significantly different (at 10\%) whereas they were significantly different in model (1) under CPT.

Let us now consider ambiguity. We find that farmers are ambiguity averse in the gain domain and ambiguity seeking in the loss domain, with $\rho<1$.

Regarding distortion of the distribution of probabilities, we find that farmers have an inverse S-shaped distribution distortion in gains with $\phi^{+}<1$, but an S-shaped distribution distortion in losses with $\phi^{-}>1$ in both models. However, results on this parameter for losses differ if we exclude from the sample subjects that made at least two switchings in at least one of the 9 series (see Appendix A.6). Numerous studies have been faced with multiple switching (Harrison et al., 2005a; Drichoutis and Nayga, 2013). There are several ways to reduce the impact of this behavior (Andersen et al., 2006; Bruner, 2011). We did not impose any restrictions on switching and we check ex post the impact of this design. In Appendix A.6, we compare parameters estimated using the whole sample vs parameters estimated excluding subjects that made at least two switchings in at least one of the 9 series. We find similar overall estimated parameters (only the magnitude slightly differs) except for distribution distorsion in losses $\phi^{-}$. In the restricted sample, subjects have an inverse $\mathrm{S}$-shaped function for distributions in losses $\phi^{-}<1$.

If we turn back to model (2) in Table 4, the probability weighting functions in the gain and loss domains are significantly different, $\phi^{+}<\phi^{-}$. Together with the previous result regarding $\gamma^{+}$and $\gamma^{-}$, this means that the effect that was captured in the risk dimension under CPT is now attributed to ambiguity under the SOM. To our knowledge, no other study has estimated distribution distortion making a distinction between gains and losses. Our results suggest that farmers' preferences about distributions involving only gains are different from their preferences about distributions involving only losses.

Finally, we find that the risk preference parameter $\alpha$ is significantly different from the ambiguity preference parameter $\rho$. These two parameters can be directly compared because our protocol involves both risky situations without ambiguity as well as ambiguous situations without risk, and because we use the same functional specification for risk and ambiguity in our models. Finally, we find that probability distortion $\gamma$ is significantly different from distribution distortion $\phi$. This significant difference validates the need for our current extension of Chakravarty and Roy (2009), who assumed these parameters to be the same. This issue has also been raised by Andersen et al. (2009).

Our estimated parameters under risk are similar to those obtained in the previous section under CPT (See section 4.1 for review of results in the literature). Concerning the estimated parameters under ambiguity, when comparing estimates, we should be as caution as we were with risk. Using students and the same specification, Chakravarty and Roy (2009) estimate an ambiguity aversion parameter of around 0.99 while we find a $\rho$ of 0.722. Barham et al. (2014) use a sample of farmers (corn and soybean, in Minnesota and Wisconsin) but with a different specification for the value function and without ML estimation. They find a value of 0.79 for ambiguity aversion. As for distribution distortion, we have not found any other similar studies for comparison. 


\section{Discussion}

Agricultural economists have considered risk for a long time. Indeed, risk aversion, loss aversion and probability distortion have been shown to play an important role for crop insurance, contract design, market finance and innovation adoption (see Bocquého et al. (2014) for a detailed review). Our results on risk preferences thus contributes to shed light on these topical issues. Less attention has been given to ambiguity so far. To the extent that decisions in agriculture involve risk and ambiguity, we need a better understanding of risk and ambiguity preferences of decision makers. The experimental elicitation of these preferences gives fruitful insights. In this article, we proposed a protocol for eliciting risk and ambiguity preferences. Our results suggest different behaviors for French farmers in the gain vs. in the loss domains, and in risky vs. in ambiguous situations. This is particularly related to the fact that French farmers distort objective probabilities in a different way when they are proposed lotteries involving financial gains or losses, and when lotteries are risky or ambiguous. More specifically, we show that farmers exhibit an inverse S-shaped distortion of first-order probabilities and of second-order probabilities except in some models for losses where second-order probabilities are S-shaped.

From a policy perspective, our results highlight the importance of considering the differences that may exist across gain and loss domains and across risky and ambiguous situations, as these differences could have specific policy implications. For example, in the risk domain, Babcock (2015) uses parameters elicited under cumulative prospect theory to explain the deductible choices of US farmers. Similar analyses could be done with respect to ambiguity using simulation or theory. With respect to pesticide use, for example, policy makers could consider the possibility that farmers have only limited knowledge about the probability of a pest attack and that they focus above all on avoiding crop loss, i.e. downside risk. In this case, policy and research efforts should be directed towards understanding farmer preferences for ambiguity in the loss domain rather than risk preferences in the gain domain, which has been the strategy used to date in the literature on pesticide issues. In this way, separable risk and ambiguity preferences can shape policy options in tangible ways. In the agricultural context, risk can be managed with insurance, whereas ambiguity could be better managed by improving the quality of information provided to farmers in order to help them more accurately appraise relevant event probabilities.

In recent years, there has been an increasing interest for ambiguity in the agricultural economics literature in various areas such as new technology adoption (Warnick et al., 2011; Barham et al., 2014), agricultural insurance (Frechette, 2000; Coble and Barnett, 2013; Skees, 1999), environmental management (Chevé and Congar, 2000; Hansen and Sargent, 2001; Roseta-Palma and Xepapadeas, 2004; Woodward and Shaw, 2008) and food safety (Chambers and Melkonyan, 2007; Melkonyan and Schubert, 2009; Chambers and Melkonyan, 2013). Regarding new technology adoption, authors have experimentally elicited ambiguity preferences to determine in a second step their effect on farmers' technology choices. In the other domains, authors either have modeled ambiguity preferences in a theoretical model or have invoked ambiguity preferences to interpret results. In the end, whether risk or ambiguity will play a role in a specific area remains an empirical question so that our results should incite researchers to consider both aspects of risk and ambiguity preferences in relation with the domain under consideration.

Consider for example new technology adoption. Objective probabilities are often difficult or impossible to establish. Since information regarding new technologies could be 
lacking, and because of uncertain benefits, adoption is directly related to risk and ambiguity preferences. Two studies have investigated the link between risk and ambiguity preferences and adoption of new technologies by farmers. In Peru, Warnick et al. (2011) have studied the impact of risk and ambiguity preferences on crop diversification. They find that ambiguity aversion leads to a lower adoption of a new crop variety (with which farmers are unfamiliar) while risk preferences have no impact. In the US, Barham et al. (2014) report that ambiguity aversion speeds up the adoption of GM corn but has no impact on the timing of adoption of GM soy. The authors explain that this may be due to GM corn including herbicide-tolerant and insect-resistant trait while GM soy only includes the herbicide-tolerant trait. The spread of weeds is assumed easier to predict for farmers than pest dynamics. Therefore, herbicide-tolerant traits are assumed to have a smaller impact on ambiguity than insect-resistant traits. According to the authors, this may explain why ambiguity aversion speeds up the adoption of GM corn. With respect to this literature, our results show that two additional parameters not considered in these articles may empirically play a role. Indeed, both articles experimentally elicit ambiguity preferences but neither protocols has allowed the elicitation of preferences in the loss domain (the considered lotteries involved only gains) and of probability distortion (all probabilities were set to $1 / 2$ ), which may each affect technology adoption. First, although positive, yields for the new technology can be perceived as losses by farmers. Farmers may have in mind a reference yield based on expectations. If the reference yield is higher than yields obtained with the new technology, farmers may perceive the technology as involving losses. Second, farmers may also be pessimistic about the distribution of yields in case of a new crop variety or of a pest attack, over-weighting the unfavorable distribution of yields and under-weighting the favorable distribution. Our results for France contribute to the literature by suggesting the need to assess carefully how a new technology will affect the risk and the ambiguity domain but also the gain and loss domain.

\section{Conclusion}

In this article, we have presented the results of a field experiment involving real payments for lottery choices designed to elicit farmers' risk and ambiguity preferences. We have used a protocol adapted from the elicitation methods proposed by Tanaka et al. (2010) for risk and Chakravarty and Roy (2009) for ambiguity on a sample of 197 French farmers. We have estimated farmers' risk and ambiguity preferences using structural models corresponding to three theoretical utility models, namely expected utility theory, cumulative prospect theory and a second-order model of ambiguity. Our results are threefold. First, we find that farmers are risk and ambiguity averse. Second, farmers are loss averse. Third, probability distortion significantly differs when facing gains vs. losses and when facing risky vs. ambiguous situations. These results highlight the importance of distinguishing between risk and ambiguity but also between gains and losses when considering farmer preferences.

Our study relies on recent advances in experimental economics allowing a better understanding of farmer behaviour in the context of risk and ambiguity. Several challenges remain concerning this area of study. Behaviour surrounding true losses is particularly difficult to study experimentally, as it is unreasonable to ask participants to pay the experimenter if the lottery involves a loss. We resolve this issue by offering participants a show-up fee that compensates for any losses incurred during the course of the experiment. 
This strategy, however, is not entirely satisfactory because it relies on losses from an artificial, experimentally-induced account rather than actual farm earnings. Another challenge is the presence of multiple switching points in the multiple price list. In our sample, $40 \%$ of farmers exhibited multiple switching points. Numerous studies have been faced with this problem. In Harrison et al. (2005a), 10 to $16 \%$ of subjects display multiple switching points and up to $85 \%$ of subjects display this behaviour in Drichoutis and Nayga (2013). There are numerous methods to reduce the impact of this behaviour, including imposing on subjects a single switching point, adding an indifference option (Andersen et al., 2006) or spending additional time explaining the lottery trade-offs to subjects (Bruner, 2011). We chose to allow our subjects to decide as freely as possible with respect to lottery choices, as we believe this approach most closely approximates the characteristics of real life decisions. For this reason, we did not impose any restrictions on switching. Despite the prevalence of this issue, there is no consensus in the literature regarding how best to deal with multiple switching points. Finally, there exist several ways to model risk and ambiguity. Most models of risk are now well documented. For ambiguity, however, several structural models are still being developed, and there is as of yet no consensus on the most appropriate model. The choice of a SOM has been criticized by Machina (2009), but an in-depth discussion of the best ambiguity model is outside the scope of the present work.

The parameters we have estimated in this study rely on strong structural modeling to ensure internal validity. Another relevant issue is thus the predictive power of the estimated parameters. There is a growing literature that addresses the ability of experimentally-elicited preferences to explain economic choices, the results of which are mixed. This is due to a number of reasons: adding context to the task has been found to increase the predictive power of elicited preferences (Menapace et al., 2016), and stakes must be sufficiently high in order for subjects to invest enough cognitive effort in the decisions and escape Rabin's critique regarding risk attitudes (Rabin, 2000). Finally, controlling for background risk is necessary since subjects make decisions in the risky experimental tasks (foreground risk) in conjunction with risky decisions made in the field (background risk) (Harrison et al., 2007). In our study, we have used a context-free task for risk attitude elicitation (context-free lotteries). Given our relatively well-off subject pool, valid concerns could be raised regarding the level of the stakes we use. Finally, as in many other experimental studies, we did not control for the level of background risk. A deeper examination of the external validity of experimentally-elicited preferences represents a challenging but necessary task.

\section{References}

Abdellaoui, M., Bleichrodt, H. and L'Haridon, O. (2008). A tractable method to measure utility and loss aversion under prospect theory. Journal of Risk and Uncertainty 36: 245-266.

Abdellaoui, M., Bleichrodt, H., L'Haridon, O. and Dolder, D. van (2016). Measuring loss aversion under ambiguity: A method to make prospect theory completely observable. Journal of Risk and Uncertainty 52: 1-20.

Andersen, S., Fountain, J., Harrison, G. W. and Rutström, E. E. (2009). Estimating Aversion to Uncertainty. Working Paper 07-2009, Copenhagen Business School, Department of Economics. 
Andersen, S., Harrison, G. W., Lau, M. I. and Rutström, E. (2010). Behavioral econometrics for psychologists. Journal of Economic Psychology 31: 553-576.

Andersen, S., Harrison, G. W., Lau, M. I. and Rutström, E. E. (2006). Elicitation using multiple price list formats. Experimental Economics 9: 383-405.

Babcock, B. A. (2015). Using cumulative prospect theory to explain anomalous crop insurance coverage choice. American Journal of Agricultural Economics .

Barberis, N. C. (2013). Thirty years of prospect theory in economics: A review and assessment. Journal of Economic Perspectives 27: 173-96.

Barham, B. L., Chavas, J.-P., Fitz, D., Salas, V. R. and Schechter, L. (2014). The roles of risk and ambiguity in technology adoption. Journal of Economic Behavior \& Organization 97: 204-218.

Binswanger, H. P. (1980). Attitudes toward risk: Experimental measurement in rural india. American Journal of Agricultural Economics 62: 395-407.

Bocquého, G., Jacquet, F. and Reynaud, A. (2014). Expected utility or prospect theory maximisers? assessing farmers' risk behaviour from field-experiment data. European Review of Agricultural Economics 41: 135-172.

Bruner, D. M. (2011). Multiple switching behaviour in multiple price lists. Applied Economics Letters 18: 417-420.

Cárdenas, J. C., De Roux, N., Jaramillo, C. R. and Martinez, L. R. (2014). Is it my money or not? An experiment on risk aversion and the house-money effect. Experimental Economics 17: 47-60.

Chakravarty, S. and Roy, J. (2009). Recursive expected utility and the separation of attitudes towards risk and ambiguity: An experimental study. Theory and Decision 66: 199-228.

Chambers, R. G. and Melkonyan, T. A. (2007). Pareto optimal trade in an uncertain world: Gmos and the precautionary principle. American Journal of Agricultural Economics 89: 520-532.

Chambers, R. G. and Melkonyan, T. A. (2013). Food scares in an uncertain world. Journal of the European Economic Association 11: 1432-1456.

Chavas, J. P., Chambers, R. G. and Pope, R. D. (2010). Production economics and farm management: A century of contributions. American Journal of Agricultural Economics 92: $356-375$.

Chevé, M. and Congar, R. (2000). Optimal pollution control under imprecise environmental risk and irreversibility. Risk Decision and Policy 5: 151-164.

Coble, K. H. and Barnett, B. J. (2013). Why do we subsidize crop insurance? American Journal of Agricultural Economics 95: 498-504.

Drichoutis, A. C. and Nayga, R. M. (2013). Eliciting risk and time preferences under induced mood states. The Journal of Socio-Economics 45: 18-27.

Ellsberg, D. (1961). Risk, ambiguity, and the savage axioms. The Quarterly Journal of Economics 75: 643-669. 
Frechette, D. L. (2000). The demand for hedging and the value of hedging opportunities. American Journal of Agricultural Economics 82: 897-907.

Galarza, F. B. (2009). Choices under Risk in Rural Peru. Staff Paper Series 542, University of Wisconsin, Agricultural and Applied Economics.

Hansen, L. and Sargent, T. J. (2001). Robust control and model uncertainty. American Economic Review 91: 60-66.

Harrison, G., List, J. and Towe, C. (2007). Naturally occurring preferences and exogenous laboratory experiments: A case study of risk aversion. Econometrica 75: 433-458.

Harrison, G. and Rutström, E. (2008). Risk aversion in the laboratory. In Cox, J. and Harrison, G. (eds), Risk Aversion in Experiments. Bingley: Emerald, Research in Experimental Economics, 41-196.

Harrison, G. and Rutström, E. (2009). Expected utility theory and prospect theory: One wedding and a decent funeral. Experimental Economics 12: 133-158.

Harrison, G. W. (2011). Experimental methods and the welfare evaluation of policy lotteries. European Review of Agricultural Economics 38: 335-360.

Harrison, G. W., Humphrey, S. J. and Verschoor, A. (2010). Choice under uncertainty: Evidence from ethiopia, india and uganda. The Economic Journal 120: 80-104.

Harrison, G. W., Johnson, E., McInnes, M. M. and Rutström, E. E. (2005a). Risk aversion and incentive effects: Comment. The American Economic Review 95: 897-901.

Harrison, G. W., Lau, M. I., Rutström, E. E. and Sullivan, M. B. (2005b). Eliciting risk and time preferences using field experiments: Some methodological issues. In Carpenter, J. P., Harrison, G. W. and List, J. A. (eds), Research in Experimental Economics, 10, $125-218$.

Harrison, G. W. and List, J. A. (2004). Field experiments. Journal of Economic Literature 42: 1009-1055.

Hellerstein, D., Higgins, N. and Horowitz, J. (2013). The predictive power of risk preference measures for farming decisions. European Review of Agricultural Economics 40: 807-833.

Holt, C. A. and Laury, S. K. (2002). Risk aversion and incentive effects. American Economic Review 92: 1644-1655.

Humphrey, S. J. and Verschoor, A. (2004). Decision-making under risk among small farmers in east uganda. Journal of African Economies 13: 44-101.

Just, R. E. and Pope, R. D. (2003). Agricultural risk analysis: Adequacy of models, data, and issues. American Journal of Agricultural Economics 85: 1249-1256.

Kahneman, D. and Tversky, A. (1979). Prospect theory: An analysis of decision under risk. Econometrica 47: 263-291.

Klibanoff, P., Marinacci, M. and Mukerji, S. (2005). A smooth model of decision making under ambiguity. Econometrica 73: 1849-1892.

Knight, F. H. (1921). Risk, Uncertainty and Profit. New York: Augustus M.Kelley. 
Liebenehm, S. and Waibel, H. (2014). Simultaneous estimation of risk and time preferences among small-scale cattle farmers in west africa. American Journal of Agricultural Economics 96: 1420-1438.

Liu, E. M. (2013). Time to change what to sow: Risk preferences and technology adoption decisions of cotton farmers in china. Review of Economics and Statistics 95: 13861403.

Loomes, G., Moffatt, P. G. and Sugden, R. (2002). A microeconometric test of alternative stochastic theories of risky choice. Journal of Risk and Uncertainty 24: 103-130.

Machina, M. J. (2009). Risk, ambiguity, and the rank-dependence axioms. American Economic Review 99: 385-92.

Melkonyan, T. A. and Schubert, J. (2009). Food safety regulations under ambiguity. American Journal of Agricultural Economics 91: 1389-1396.

Menapace, L., Colson, G. and Raffaelli, R. (2016). A comparison of hypothetical risk attitude elicitation instruments for explaining farmer crop insurance purchases. European Review of Agricultural Economics 43: 113-135.

Moschini, G. and Hennessy, D. A. (2001). Uncertainty, risk aversion, and risk management for agricultural producers. In Gardner, B. and Rausser, G. (eds), Handbook of Agricultural Economics. Elsevier Science B.V, Volume 1, 87-153.

Nau, R. F. (2006). Uncertainty aversion with second-order utilities and probabilities. Management Science 52: 136-145.

Neumann, J. von and Morgenstern, O. (1947). Theory of games and economic behavior. Princeton University Press.

Nguyen, Q. (2011). Does nurture matter: theory and experimental investigation on the effect of working environment on risk and time preferences. Journal of Risk and Uncertainty 43: 245-270.

Nguyen, Q. and Leung, P. (2010). How nurture can shape preferences: an experimental study on risk preferences of vietnamese fishers. Environment and Development Economics 15: 609-631.

Prelec, D. (1998). The probability weighting function. Econometrica 66: 497-527.

Rabin, M. (2000). Risk aversion and expected-utility theory: A calibration theorem. Econometrica 68: 1281-1292.

Reynaud, A. and Couture, S. (2012). Stability of risk preference measures: results from a field experiment on french farmers. Theory and Decision 73: 203-221.

Roseta-Palma, C. and Xepapadeas, A. (2004). Robust control in water management. Journal of Risk and Uncertainty 29: 21-34.

Schmidt, U. and Zank, H. (2005). What is Loss Aversion? Journal of Risk and Uncertainty 30: 157-167.

Skees, J. R. (1999). Opportunities for improved efficiency in risk sharing using capital markets. American Journal of Agricultural Economics 81: 1228-1233. 
Tanaka, T., Camerer, C. F. and Nguyen, Q. (2010). Risk and time preferences: Linking experimental and household survey data from vietnam. American Economic Review 100: $557-571$.

Trujillo-Barrera, A., Pennings, J. M. E. and Hofenk, D. (2016). Understanding producers' motives for adopting sustainable practices: the role of expected rewards, risk perception and risk tolerance. European Review of Agricultural Economics .

Tversky, A. and Kahneman, D. (1992). Advances in prospect-theory-cumulative representation of uncertainty. Journal of Risk and Uncertainty 5: 297-323.

Warnick, J. C. E., Laszlo, S. C. and Escobal, J. (2011). Ambiguity aversion and portfolio choice in small-scale peruvian farming. The B. E . Journal of Economic Analysis \& Policy Volume 11.

Woodward, R. T. and Shaw, W. D. (2008). Allocating resources in an uncertain world: Water management and endangered species. American Journal of Agricultural Economics 90: 593-605. 


\section{A Appendix}

\section{A.1 Questionnaire (translated from French)}

You are going to participate to an experimental survey. You must choose between two options, A and B, in 9 series of choice sets. Each option is called a lottery or an urn. Each lottery involves gains or losses with a given probability. You may earn money according to the choices you make in the experiment. At the end of the survey, one choice set among all of the choice sets in the 9 series will be drawn at random, and the lottery you chose in that particular choice set will be played. This will determine the amount you will earn or lose. Please note that the posted amounts will be divided by 50 .

\section{A.1.1 Series \#1 (Series RG1)}

In this series, you will make several binary lottery choices. Let us take an example. Consider the following choice set.

Choose the lottery you prefer:

\begin{tabular}{|c|c|c|c|c|}
\hline \multicolumn{2}{|c|}{ Urn A } & \multicolumn{2}{c|}{ Urn B } & \multirow{2}{*}{ Choice } \\
3 WHITE + 7 RED & \multicolumn{2}{c|}{1 WHITE + 9 RED } & \\
\cline { 1 - 3 } $\begin{array}{c}\text { If drawn ball is } \\
\text { WHITE }\end{array}$ & $\begin{array}{c}\text { If drawn ball is } \\
\text { RED }\end{array}$ & $\begin{array}{c}\text { If drawn ball is } \\
\text { WHITE }\end{array}$ & $\begin{array}{c}\text { If drawn ball is } \\
\text { RED }\end{array}$ & \\
\cline { 1 - 4 } $3 / 10$ & $7 / 10$ & $1 / 10$ & $9 / 10$ & \\
\hline $400 €$ & $100 €$ & $680 €$ & $50 €$ & A \\
\hline
\end{tabular}

Each urn contains 10 balls.

- Urn A contains 3 WHITE balls and 7 RED balls. If a WHITE ball is drawn (3 times out of 10), you will earn an outcome of 400; but if a RED ball is drawn (7 times out of 10), you will earn an outcome of 100.

- Urn B contains 1 WHITE ball and 9 RED balls. If a WHITE ball is drawn (1 time out of 10), you will earn an outcome of 680; but if a RED ball is drawn (9 times out of 10), you will earn an outcome of 50 .

You will make several choices between two urns that are similar to the urns in the example presented above. Do you have any questions before we begin?

Let us begin. Consider the following choice sets.

\section{A.1.2 Series \#2 (Series RG2)}

In this series, you are going to make several binary lottery choices. Note that the probabilities and amounts have changed.

\section{A.1.3 Series \#3 (Series RGL)}

In this series, you are going to make several binary lottery choices. Note that the probabilities and amounts have changed. Note also that amounts can involve gains or losses. 
For each row, choose which lottery you prefer:

\begin{tabular}{|c|c|c|c|cr|}
\hline \multicolumn{2}{|c|}{ Urn A } & \multicolumn{2}{c|}{ Urn B } & Choice \\
\hline $3 / 10$ & $7 / 10$ & $1 / 10$ & $9 / 10$ & & \\
\hline $200 €$ & $100 €$ & $270 €$ & $75 €$ & $\mathrm{~A}$ & $\mathrm{~B}$ \\
\hline $200 €$ & $100 €$ & $280 €$ & $75 €$ & $\mathrm{~A}$ & $\mathrm{~B}$ \\
\hline $200 €$ & $100 €$ & $350 €$ & $75 €$ & $\mathrm{~A}$ & $\mathrm{~B}$ \\
\hline $200 €$ & $100 €$ & $390 €$ & $75 €$ & $\mathrm{~A}$ & $\mathrm{~B}$ \\
\hline $200 €$ & $100 €$ & $430 €$ & $75 €$ & $\mathrm{~A}$ & $\mathrm{~B}$ \\
\hline $200 €$ & $100 €$ & $450 €$ & $75 €$ & $\mathrm{~A}$ & $\mathrm{~B}$ \\
\hline $200 €$ & $100 €$ & $480 €$ & $75 €$ & $\mathrm{~A}$ & $\mathrm{~B}$ \\
\hline $200 €$ & $100 €$ & $520 €$ & $75 €$ & $\mathrm{~A}$ & $\mathrm{~B}$ \\
\hline $200 €$ & $100 €$ & $600 €$ & $75 €$ & $\mathrm{~A}$ & $\mathrm{~B}$ \\
\hline $200 €$ & $100 €$ & $700 €$ & $75 €$ & $\mathrm{~A}$ & $\mathrm{~B}$ \\
\hline $200 €$ & $100 €$ & $900 €$ & $75 €$ & $\mathrm{~A}$ & $\mathrm{~B}$ \\
\hline $200 €$ & $100 €$ & $1,200 €$ & $75 €$ & $\mathrm{~A}$ & $\mathrm{~B}$ \\
\hline $200 €$ & $100 €$ & $2,200 €$ & $75 €$ & $\mathrm{~A}$ & $\mathrm{~B}$ \\
\hline $200 €$ & $100 €$ & $3,000 €$ & $75 €$ & $\mathrm{~A}$ & $\mathrm{~B}$ \\
\hline
\end{tabular}

Table A.1: Series RG1

For each row, choose which lottery you prefer:

\begin{tabular}{|c|c|c|c|cc|}
\hline \multicolumn{2}{|c|}{ Urn A } & \multicolumn{2}{c|}{ Urn B } & \multirow{2}{*}{ Choice } \\
\hline $9 / 10$ & $1 / 10$ & $7 / 10$ & $3 / 10$ & & \\
\hline $400 €$ & $300 €$ & $450 €$ & $50 €$ & $\mathrm{~A}$ & $\mathrm{~B}$ \\
\hline $400 €$ & $300 €$ & $485 €$ & $50 €$ & $\mathrm{~A}$ & $\mathrm{~B}$ \\
\hline $400 €$ & $300 €$ & $500 €$ & $50 €$ & $\mathrm{~A}$ & $\mathrm{~B}$ \\
\hline $400 €$ & $300 €$ & $525 €$ & $50 €$ & $\mathrm{~A}$ & $\mathrm{~B}$ \\
\hline $400 €$ & $300 €$ & $540 €$ & $50 €$ & $\mathrm{~A}$ & $\mathrm{~B}$ \\
\hline $400 €$ & $300 €$ & $560 €$ & $50 €$ & $\mathrm{~A}$ & $\mathrm{~B}$ \\
\hline $400 €$ & $300 €$ & $580 €$ & $50 €$ & $\mathrm{~A}$ & $\mathrm{~B}$ \\
\hline $400 €$ & $300 €$ & $600 €$ & $50 €$ & $\mathrm{~A}$ & $\mathrm{~B}$ \\
\hline $400 €$ & $300 €$ & $625 €$ & $50 €$ & $\mathrm{~A}$ & $\mathrm{~B}$ \\
\hline $400 €$ & $300 €$ & $640 €$ & $50 €$ & $\mathrm{~A}$ & $\mathrm{~B}$ \\
\hline $400 €$ & $300 €$ & $680 €$ & $50 €$ & $\mathrm{~A}$ & $\mathrm{~B}$ \\
\hline $400 €$ & $300 €$ & $740 €$ & $50 €$ & $\mathrm{~A}$ & $\mathrm{~B}$ \\
\hline $400 €$ & $300 €$ & $820 €$ & $50 €$ & $\mathrm{~A}$ & $\mathrm{~B}$ \\
\hline $400 €$ & $300 €$ & $1,000 €$ & $50 €$ & $\mathrm{~A}$ & $\mathrm{~B}$ \\
\hline
\end{tabular}

Table A.2: Series RG2

\section{A.1.4 Series \#4 (Series RL1)}

In this series, you are going to make several binary lottery choices. Note that the probabilities and amounts have changed. Note also that amounts involve losses.

\section{A.1.5 Series \#5 (Series RL2)}

In this series, you are going to make several binary lottery choices. Note that the probabilities and amounts have changed. Note also that amounts still involve losses. 
For each row, choose which lottery you prefer:

\begin{tabular}{|c|c|c|c|cr|}
\hline \multicolumn{2}{|c|}{ Urn A } & \multicolumn{2}{c|}{ Urn B } & \multirow{2}{*}{ Choice } \\
\hline $5 / 10$ & $5 / 10$ & $5 / 10$ & $5 / 10$ & & \\
\hline $250 €$ & $-40 €$ & $300 €$ & $-210 €$ & A & B \\
\hline $40 €$ & $-40 €$ & $300 €$ & $-210 €$ & A & B \\
\hline $10 €$ & $-40 €$ & $300 €$ & $-210 €$ & A & B \\
\hline $10 €$ & $-40 €$ & $300 €$ & $-160 €$ & A & B \\
\hline $10 €$ & $-80 €$ & $300 €$ & $-160 €$ & A & B \\
\hline $10 €$ & $-80 €$ & $300 €$ & $-140 €$ & A & B \\
\hline $10 €$ & $-80 €$ & $300 €$ & $-110 €$ & A & B \\
\hline
\end{tabular}

Table A.3: Series RGL

For each row, choose which lottery you prefer:

\begin{tabular}{|c|c|c|c|l|l|}
\hline \multicolumn{2}{|c|}{ Urn A } & \multicolumn{2}{c|}{ Urn B } & \multirow{2}{*}{ Choice } \\
\hline $1 / 10$ & $9 / 10$ & $3 / 10$ & $7 / 10$ & & \\
\hline$-200 €$ & $-75 €$ & $-150 €$ & $-100 €$ & $\mathrm{~A}$ & $\mathrm{~B}$ \\
\hline$-210 €$ & $-75 €$ & $-150 €$ & $-100 €$ & $\mathrm{~A}$ & $\mathrm{~B}$ \\
\hline$-350 €$ & $-75 €$ & $-150 €$ & $-100 €$ & $\mathrm{~A}$ & $\mathrm{~B}$ \\
\hline$-390 €$ & $-75 €$ & $-150 €$ & $-100 €$ & $\mathrm{~A}$ & $\mathrm{~B}$ \\
\hline$-430 €$ & $-75 €$ & $-150 €$ & $-100 €$ & $\mathrm{~A}$ & $\mathrm{~B}$ \\
\hline$-480 €$ & $-75 €$ & $-150 €$ & $-100 €$ & $\mathrm{~A}$ & $\mathrm{~B}$ \\
\hline$-520 €$ & $-75 €$ & $-150 €$ & $-100 €$ & $\mathrm{~A}$ & $\mathrm{~B}$ \\
\hline$-540 €$ & $-75 €$ & $-150 €$ & $-100 €$ & $\mathrm{~A}$ & $\mathrm{~B}$ \\
\hline$-580 €$ & $-75 €$ & $-150 €$ & $-100 €$ & $\mathrm{~A}$ & $\mathrm{~B}$ \\
\hline$-620 €$ & $-75 €$ & $-150 €$ & $-100 €$ & $\mathrm{~A}$ & $\mathrm{~B}$ \\
\hline$-700 €$ & $-75 €$ & $-150 €$ & $-100 €$ & $\mathrm{~A}$ & $\mathrm{~B}$ \\
\hline$-780 €$ & $-75 €$ & $-150 €$ & $-100 €$ & $\mathrm{~A}$ & $\mathrm{~B}$ \\
\hline$-850 €$ & $-75 €$ & $-150 €$ & $-100 €$ & $\mathrm{~A}$ & $\mathrm{~B}$ \\
\hline$-1,000 €$ & $-75 €$ & $-150 €$ & $-100 €$ & $\mathrm{~A}$ & $\mathrm{~B}$ \\
\hline
\end{tabular}

Table A.4: Series RL1

\section{A.1.6 Series \#6 (Series AG1)}

In this series, you are going to make several binary lottery choices. Note, however, that the game has changed. Urn A and urn B still contain 10 balls each.

- Urn A contains 5 WHITE balls and 5 RED balls.

- Urn B contains 10 balls that are either all WHITE or all RED. You do not know the colour of the balls in urn B. But you do know that the 10 balls in urn B are of the same colour.

You will be asked to choose which urn you prefer and which colour you want to bet on, WHITE or RED. If urn A or urn B is played for payments, the colour you bet on will be compared to the colour of the ball that is drawn to determine the amount that you earn.

Note that given that you choose the colour, the experimenter has no incentive to choose one colour over another for urn B.

In the following example, suppose you bet on RED (you circled RED on the first line).

- Suppose further that you chose urn A. If the drawn ball is RED (the colour you bet on), you earn 1,000. But if the drawn ball is WHITE, you earn 0 . 
For each row, choose which lottery you prefer:

\begin{tabular}{|c|l|l|l|ll|}
\hline \multicolumn{2}{|c|}{ Urn A } & \multicolumn{2}{c|}{ Urn B } & \multirow{2}{*}{ Choice } \\
\hline $7 / 10$ & $3 / 10$ & $9 / 10$ & $1 / 10$ & & \\
\hline$-450 €$ & $-50 €$ & $-400 €$ & $-300 €$ & $\mathrm{~A}$ & $\mathrm{~B}$ \\
\hline$-480 €$ & $-50 €$ & $-400 €$ & $-300 €$ & $\mathrm{~A}$ & $\mathrm{~B}$ \\
\hline$-490 €$ & $-50 €$ & $-400 €$ & $-300 €$ & $\mathrm{~A}$ & $\mathrm{~B}$ \\
\hline$-500 €$ & $-50 €$ & $-400 €$ & $-300 €$ & $\mathrm{~A}$ & $\mathrm{~B}$ \\
\hline$-510 €$ & $-50 €$ & $-400 €$ & $-300 €$ & $\mathrm{~A}$ & $\mathrm{~B}$ \\
\hline$-525 €$ & $-50 €$ & $-400 €$ & $-300 €$ & $\mathrm{~A}$ & $\mathrm{~B}$ \\
\hline$-540 €$ & $-50 €$ & $-400 €$ & $-300 €$ & $\mathrm{~A}$ & $\mathrm{~B}$ \\
\hline$-560 €$ & $-50 €$ & $-400 €$ & $-300 €$ & $\mathrm{~A}$ & $\mathrm{~B}$ \\
\hline$-580 €$ & $-50 €$ & $-400 €$ & $-300 €$ & $\mathrm{~A}$ & $\mathrm{~B}$ \\
\hline$-600 €$ & $-50 €$ & $-400 €$ & $-300 €$ & $\mathrm{~A}$ & $\mathrm{~B}$ \\
\hline$-625 €$ & $-50 €$ & $-400 €$ & $-300 €$ & $\mathrm{~A}$ & $\mathrm{~B}$ \\
\hline$-640 €$ & $-50 €$ & $-400 €$ & $-300 €$ & $\mathrm{~A}$ & $\mathrm{~B}$ \\
\hline$-800 €$ & $-50 €$ & $-400 €$ & $-300 €$ & $\mathrm{~A}$ & $\mathrm{~B}$ \\
\hline$-1,000 €$ & $-50 €$ & $-400 €$ & $-300 €$ & $\mathrm{~A}$ & $\mathrm{~B}$ \\
\hline
\end{tabular}

Table A.5: Series RL2

- Now suppose that you chose urn B. If the drawn ball is RED (the colour you bet on), you earn 100. But if the drawn ball is WHITE, you earn 0.

Choose which colour you want to bet on: WHITE or RED. Circle your choice:

$\mathrm{W}$

(R)

Then for each row, choose which lottery you prefer:

\begin{tabular}{|c|c|c|c|c|}
\hline \multicolumn{2}{|c|}{ Urn A } & \multicolumn{2}{c|}{$\begin{array}{c}\text { Urn B } \\
\text { 10 Balls of same colour } \\
(10 \text { WHITE or 10 RED })\end{array}$} & \multirow{2}{*}{ Choice } \\
\cline { 1 - 4 } $\begin{array}{c}\text { If you don't pick } \\
\text { a ball of the } \\
\text { colours you bet on }\end{array}$ & $\begin{array}{c}\text { If you pick } \\
\text { a ball of the } \\
\text { colour you bet on }\end{array}$ & $\begin{array}{c}\text { If you don't pick } \\
\text { a ball of the } \\
\text { colours you bet on }\end{array}$ & $\begin{array}{c}\text { If you pick } \\
\text { a ball of the } \\
\text { colour you bet on }\end{array}$ & \\
\hline $0 €$ & $1,000 €$ & $0 €$ & $100 €$ & A \\
\hline \hline
\end{tabular}


Below is a series of lottery choices. Please answer the following questions based on the series below.

1. Suppose that you bet on RED and chose urn B on line 9. Suppose further that line 9 is randomly drawn to be played for payment. What will be your gain if urn B contains:

- a. WHITE balls?

- b. RED balls?

2. Suppose that you bet on WHITE. After a line was randomly selected for payment, you earned 30. Can you indicate which line was randomly drawn to be played for payment?

3. Suppose that you bet on a colour and that line 7 is randomly selected for payment. Suppose further that you chose urn B at line 7 and that urn B contained RED balls. Which colour did you bet on?

Do you have any questions before we begin? 
Choose which colour you want to bet on: WHITE or RED. Circle your choice:

$\mathrm{W}$

$\mathrm{R}$

Then for each row, choose which lottery you prefer.

\begin{tabular}{|c|c|c|c|c|}
\hline \multicolumn{2}{|c|}{$\begin{array}{c}\text { Urn A } \\
5 \text { WHITE + 5 RED }\end{array}$} & \multicolumn{2}{|c|}{$\begin{array}{c}\text { Urn B } \\
\text { 10 Balls of the same colour } \\
\text { (10 WHITE or } 10 \text { RED) }\end{array}$} & \multirow[t]{2}{*}{ Choice } \\
\hline $\begin{array}{l}\text { If you don't pick } \\
\text { a ball of the } \\
\text { colours you bet on }\end{array}$ & $\begin{array}{c}\text { If you pick } \\
\text { a ball of the } \\
\text { colour you bet on }\end{array}$ & $\begin{array}{l}\text { If you don't pick } \\
\text { a ball of the } \\
\text { colours you bet on }\end{array}$ & $\begin{array}{c}\text { If you pick } \\
\text { a ball of the } \\
\text { colour you bet on }\end{array}$ & \\
\hline $0 €$ & $1000 €$ & $0 €$ & $100 €$ & A \\
\hline $0 €$ & $750 €$ & $0 €$ & $100 €$ & A \\
\hline $0 €$ & $500 €$ & $0 €$ & $100 €$ & $\bar{A}$ \\
\hline $0 €$ & $250 €$ & $0 €$ & $100 €$ & A \\
\hline $0 €$ & $150 €$ & $0 €$ & $100 €$ & $\bar{A}$ \\
\hline $0 €$ & $110 €$ & $0 €$ & $100 €$ & A \\
\hline $0 €$ & $100 €$ & $0 €$ & $100 €$ & $\mathrm{~A}$ \\
\hline $0 €$ & $90 €$ & $0 €$ & $100 €$ & A \\
\hline $0 €$ & $70 €$ & $0 €$ & $100 €$ & $\bar{A}$ \\
\hline $0 €$ & $50 €$ & $0 €$ & $100 €$ & A \\
\hline $0 €$ & $40 €$ & $0 €$ & $100 €$ & A \\
\hline $0 €$ & $30 €$ & $0 €$ & $100 €$ & A \\
\hline $0 €$ & $20 €$ & $0 €$ & $100 €$ & A \\
\hline $0 €$ & $10 €$ & $0 €$ & $100 €$ & $\mathrm{~A}$ \\
\hline
\end{tabular}

Table A.6: Series AG1

Consider urn B. In your opinion, what is the probability that the balls in urn B are of the same colour you bet on?

\section{A.1.7 Series \#7 (Series AG2)}

In this series, you are going to make several binary lottery choices. Note, however, that the game has changed. Urn A and urn B contain 15 balls each.

- Urn A contains 5 BLUE balls, 5 YELLOW balls and 5 GREEN balls.

- Urn B contains 15 balls of the same colour, which are either all BLUE or all YELLOW or all GREEN. You do not know the colour of the balls in urn B. But you do know that the 15 balls in urn $\mathrm{B}$ are of the same colour.

You will be asked to choose which urn you prefer and choose which colour you want to bet on, BLUE, YELLOW or GREEN. If urn A or urn B is played for payment, the colour you bet on will be compared to the colour of the ball that is drawn to determine the amount you earn.

Note that given that you choose the colour, the experimenter has no incentive to choose one colour over another for urn B.

In the following example, suppose you bet on GREEN (you circled GREEN on the first line).

- Suppose further that you chose urn A. If the drawn ball is GREEN (the colour you bet on), you earn 1,050. But if the drawn ball is BLUE or YELLOW, you earn 0. 
- Now suppose that you chose urn B. If the drawn ball is GREEN (the colour you bet on), you earn 75. But if the drawn ball is BLUE or YELLOW, you earn 0.

Choose which colour you want to bet on: BLUE or YELLOW or GREEN. Circle your choice:

B $\quad$ Y G

Choose which lottery you prefer.

\begin{tabular}{|c|c|c|c|c|}
\hline \multicolumn{2}{|c|}{$\begin{array}{c}\text { Urn A } \\
5 \text { BLUE + } 5 \text { YELLOW + 5 GREEN }\end{array}$} & \multicolumn{2}{|c|}{$\begin{array}{c}\text { Urn B } \\
15 \text { Balls of the same colour } \\
\text { (BLUE or YELLOW or GREEN) }\end{array}$} & \multirow[t]{2}{*}{ Choice } \\
\hline $\begin{array}{l}\text { If you don't pick } \\
\text { a ball of the } \\
\text { colours you bet on }\end{array}$ & $\begin{array}{c}\text { If you pick } \\
\text { a ball of the } \\
\text { colour you bet on }\end{array}$ & $\begin{array}{l}\text { If you don't pick } \\
\text { a ball of the } \\
\text { colours you bet on }\end{array}$ & $\begin{array}{c}\text { If you pick } \\
\text { a ball of the } \\
\text { colour you bet on }\end{array}$ & \\
\hline $0 €$ & $1,050 €$ & $0 €$ & $75 €$ & $\mathrm{~A}$ \\
\hline
\end{tabular}

Do you have any questions before we begin?

Let us begin. Consider the following choice sets. 
Choose which colour you want to bet on: BLUE, YELLOW or GREEN. Circle your choice:

B $\quad$ Y $\quad$ G

Then for each row, choose which lottery you prefer.

\begin{tabular}{|c|c|c|c|c|}
\hline \multicolumn{2}{|c|}{$\begin{array}{c}\text { Urn A } \\
5 \text { BLUE + } 5 \text { YELLOW + } 5 \text { GREEN }\end{array}$} & \multicolumn{2}{|c|}{$\begin{array}{c}\text { Urn B } \\
15 \text { Balls of the same colour } \\
\text { (BLUE or YELLOW or GREEN) }\end{array}$} & \multirow[t]{2}{*}{ Choice } \\
\hline $\begin{array}{l}\text { If you don't pick } \\
\text { a ball of the } \\
\text { colours you bet on }\end{array}$ & $\begin{array}{c}\text { If you pick } \\
\text { a ball of the } \\
\text { colour you bet on }\end{array}$ & $\begin{array}{l}\text { If you don't pick } \\
\text { a ball of the } \\
\text { colours you bet on }\end{array}$ & $\begin{array}{c}\text { If you pick } \\
\text { a ball of the } \\
\text { colour you bet on }\end{array}$ & \\
\hline $0 €$ & $1050 €$ & $0 €$ & $75 €$ & $\mathrm{~A}$ \\
\hline $0 €$ & $500 €$ & $0 €$ & $75 €$ & $\mathrm{~A}$ \\
\hline $0 €$ & $250 €$ & $0 €$ & $75 €$ & $\mathrm{~A}$ \\
\hline $0 €$ & $100 €$ & $0 €$ & $75 €$ & $\mathrm{~A}$ \\
\hline $0 €$ & $80 €$ & $0 €$ & $75 €$ & $\mathrm{~A}$ \\
\hline $0 €$ & $70 €$ & $0 €$ & $75 €$ & $\mathrm{~A}$ \\
\hline $0 €$ & $60 €$ & $0 €$ & $75 €$ & $\mathrm{~A}$ \\
\hline $0 €$ & $50 €$ & $0 €$ & $75 €$ & $\mathrm{~A}$ \\
\hline $0 €$ & $40 €$ & $0 €$ & $75 €$ & $\mathrm{~A}$ \\
\hline $0 €$ & $35 €$ & $0 €$ & $75 €$ & $\mathrm{~A}$ \\
\hline $0 €$ & $30 €$ & $0 €$ & $75 €$ & $\mathrm{~A}$ \\
\hline $0 €$ & $25 €$ & $0 €$ & $75 €$ & $\mathrm{~A}$ \\
\hline $0 €$ & $15 €$ & $0 €$ & $75 €$ & $\mathrm{~A}$ \\
\hline $0 €$ & $5 €$ & $0 €$ & $75 €$ & $\mathrm{~A}$ \\
\hline
\end{tabular}

Table A.7: Series AG2

Consider urn B. In your opinion, what is the probability that the balls in urn B are of the same colour you bet on?

\section{A.1.8 Series \#8 (Series AL1)}

In this series, you are going to make several binary lottery choices. Note, however, that the game has changed. Urn A and urn B contain 10 balls each.

- Urn A contains 5 WHITE balls and 5 RED balls.

- Urn B contains 10 balls of the same colour, which are either all WHITE or all RED. You do not know the colour of the balls in urn B. But you do know that the 10 balls in urn B are of the same colour.

You will be asked to choose which urn you prefer and choose which colour you want to bet on, WHITE or RED. If urn A or urn B is played for payment, the colour you bet on will be compared to the colour of the ball that is drawn to determine the amount you earn.

Note that given that you choose the colour, the experimenter has no incentive to choose one colour over another for urn B.

In the following example, suppose you bet on RED (you circled RED on the first line).

- Suppose further that you chose urn A. If the drawn ball is RED (the colour you bet on), you earn 0. But if the drawn ball is WHITE, you lose 10.

- Now suppose that you chose urn B. If the drawn ball is RED (the colour you bet on), you earn 0. But if the drawn ball is WHITE, you lose 100. 
Choose which colour you want to bet on: WHITE or RED. Circle you choice.

$\mathrm{W}$

(R)

Then for each row, choose which lottery you prefer.

\begin{tabular}{|c|c|c|c|c|}
\hline \multicolumn{2}{|c|}{ Urn A } & \multicolumn{2}{c|}{$\begin{array}{c}\text { Urn B } \\
\text { 10 Balls of the same colour } \\
(10 \text { WHITE or 10 RED })\end{array}$} & \multirow{2}{*}{ Choice } \\
\cline { 1 - 4 } $\begin{array}{c}\text { If you don't pick } \\
\text { a ball of the } \\
\text { colours you bet on }\end{array}$ & $\begin{array}{c}\text { If you pick } \\
\text { a ball of the } \\
\text { colour you bet on }\end{array}$ & $\begin{array}{c}\text { If you don't pick } \\
\text { a ball of the } \\
\text { colours you bet on }\end{array}$ & $\begin{array}{c}\text { If you pick } \\
\text { a ball of the } \\
\text { colour you bet on }\end{array}$ & \\
\cline { 1 - 5 } 0 & $-10 €$ & $0 €$ & $-100 €$ & A \\
\hline
\end{tabular}

Do you have any questions before we begin? Let us begin. Consider the following choice sets. 
Choose which colour you want to bet on: WHITE or RED. Circle you choice.

$\mathrm{W}$

$\mathrm{R}$

Then for each row, choose which lottery you prefer.

\begin{tabular}{|c|c|c|c|c|c|}
\hline \multicolumn{2}{|c|}{$\begin{array}{c}\text { Urn A } \\
5 \text { WHITE + } 5 \text { RED }\end{array}$} & \multicolumn{2}{|c|}{$\begin{array}{c}\text { Urn B } \\
10 \text { Balls of the same colour } \\
\text { (10 WHITE or } 10 \text { RED })\end{array}$} & \multirow{2}{*}{\multicolumn{2}{|c|}{ Choice }} \\
\hline $\begin{array}{l}\text { If you don't pick } \\
\text { a ball of the } \\
\text { colours you bet on }\end{array}$ & $\begin{array}{c}\text { If you pick } \\
\text { a ball of the } \\
\text { colour you bet on }\end{array}$ & $\begin{array}{l}\text { If you don't pick } \\
\text { a ball of the } \\
\text { colours you bet on }\end{array}$ & $\begin{array}{c}\text { If you pick } \\
\text { a ball of the } \\
\text { colour you bet on }\end{array}$ & & \\
\hline $0 €$ & $-10 €$ & $0 €$ & $-100 €$ & A & B \\
\hline $0 €$ & $-50 €$ & $0 €$ & $-100 €$ & $\mathrm{~A}$ & B \\
\hline $0 €$ & $-75 €$ & $0 €$ & $-100 €$ & $\mathrm{~A}$ & $\bar{B}$ \\
\hline $0 €$ & $-100 €$ & $0 €$ & $-100 €$ & A & B \\
\hline $0 €$ & $-125 €$ & $0 €$ & $-100 €$ & A & B \\
\hline $0 €$ & $-150 €$ & $0 €$ & $-100 €$ & A & B \\
\hline $0 €$ & $-160 €$ & $0 €$ & $-100 €$ & A & B \\
\hline $0 €$ & $-170 €$ & $0 €$ & $-100 €$ & A & B \\
\hline $0 €$ & $-180 €$ & $0 €$ & $-100 €$ & A & B \\
\hline $0 €$ & $-200 €$ & $0 €$ & $-100 €$ & A & B \\
\hline $0 €$ & $-220 €$ & $0 €$ & $-100 €$ & A & B \\
\hline $0 €$ & $-250 €$ & $0 €$ & $-100 €$ & $\mathrm{~A}$ & B \\
\hline $0 €$ & $-500 €$ & $0 €$ & $-100 €$ & A & B \\
\hline $0 €$ & $-1,000 €$ & $0 €$ & $-100 €$ & $\mathrm{~A}$ & $\bar{B}$ \\
\hline
\end{tabular}

Table A.8: Series AL1

Consider urn B. In your opinion, what is the probability that the balls in urn B are of the same colour you bet on?

\section{A.1.9 Series \#9 (Series AL2)}

In this series, you are going to make several binary lottery choices. Note, however, that the game has changed. Urn A and urn B contain 15 balls each.

- Urn A contains 5 BLUE balls, 5 YELLOW balls and 5 GREEN balls.

- Urn B contains 15 balls of the same colour, which are either all BLUE or all YELLOW or all GREEN. You do not know the colour of the balls in urn B. But you do know that the 15 balls in urn B are of the same colour.

You will be asked to choose which urn you prefer and which colour you want to bet on, BLUE, YELLOW or GREEN. If urn A or urn B is played for payment, the colour you bet on will be compared to the colour of the ball that is drawn to determine the amount you earn.

Note that given that you choose the colour, the experimenter has no incentive to choose one colour over another for urn B.

In the following example, suppose you bet on GREEN (you circled GREEN on the first line).

- Suppose further that you chose urn A. If the drawn ball is GREEN (the colour you bet on), you earn 0. But if the drawn ball is BLUE or YELLOW, you lose 5. 
- Now suppose that you chose urn B. If the drawn ball is GREEN (the colour you bet on), you earn 0. But if the drawn ball is BLUE or YELLOW, you lose 75.

Choose which colour you want to bet on: BLUE, YELLOW or GREEN. Circle your choice:

B $\quad \mathrm{Y} \quad \mathrm{G}$

Choose which lottery you prefer.

\begin{tabular}{|c|c|c|c|c|}
\hline \multicolumn{2}{|c|}{$\begin{array}{c}\text { Urn A } \\
5 \text { BLUE + } 5 \text { YELLOW + 5 GREEN }\end{array}$} & \multicolumn{2}{|c|}{$\begin{array}{c}\text { Urn B } \\
15 \text { Balls of the same colour } \\
\text { (BLUE or YELLOW or GREEN) }\end{array}$} & \multirow[t]{2}{*}{ Choice } \\
\hline $\begin{array}{l}\text { If you don't pick } \\
\text { a ball of the } \\
\text { colours you bet on }\end{array}$ & $\begin{array}{c}\text { If you pick } \\
\text { a ball of the } \\
\text { colour you bet on }\end{array}$ & $\begin{array}{l}\text { If you don't pick } \\
\text { a ball of the } \\
\text { colours you bet on }\end{array}$ & $\begin{array}{c}\text { If you pick } \\
\text { a ball of the } \\
\text { colour you bet on }\end{array}$ & \\
\hline $0 €$ & $-5 €$ & $0 €$ & $-75 €$ & $\mathrm{~A}$ \\
\hline
\end{tabular}

Do you have any questions before we begin? Let us begin. Consider the following choice sets. 
Choose which colour you want to bet on: BLUE, YELLOW or GREEN. Circle your choice:

B $\quad \mathrm{Y} \quad \mathrm{G}$

Then for each row, choose which lottery you prefer.

\begin{tabular}{|c|c|c|c|c|}
\hline \multicolumn{2}{|c|}{$\begin{array}{c}\text { Urn A } \\
5 \text { BLUE + } 5 \text { YELLOW + 5 GREEN }\end{array}$} & \multicolumn{2}{|c|}{$\begin{array}{c}\text { Urn B } \\
15 \text { Balls of the same colour } \\
\text { (BLUE or YELLOW or GREEN) }\end{array}$} & \multirow[t]{2}{*}{ Choice } \\
\hline $\begin{array}{l}\text { If you don't pick } \\
\text { a ball of the } \\
\text { colours you bet on }\end{array}$ & $\begin{array}{c}\text { If you pick } \\
\text { a ball of the } \\
\text { colour you bet on }\end{array}$ & $\begin{array}{l}\text { If you don't pick } \\
\text { a ball of the } \\
\text { colours you bet on }\end{array}$ & $\begin{array}{c}\text { If you pick } \\
\text { a ball of the } \\
\text { colour you bet on }\end{array}$ & \\
\hline $0 €$ & $-5 €$ & $0 €$ & $-75 €$ & $\mathrm{~A}$ \\
\hline $0 €$ & $-20 €$ & $0 €$ & $-75 €$ & A \\
\hline $0 €$ & $-30 €$ & $0 €$ & $-75 €$ & $\mathrm{~A}$ \\
\hline $0 €$ & $-40 €$ & $0 €$ & $-75 €$ & A \\
\hline $0 €$ & $-50 €$ & $0 €$ & $-75 €$ & $\mathrm{~A}$ \\
\hline $0 €$ & $-60 €$ & $0 €$ & $-75 €$ & A \\
\hline $0 €$ & $-70 €$ & $0 €$ & $-75 €$ & A \\
\hline $0 €$ & $-75 €$ & $0 €$ & $-75 €$ & A \\
\hline $0 €$ & $-85 €$ & $0 €$ & $-75 €$ & A \\
\hline $0 €$ & $-100 €$ & $0 €$ & $-75 €$ & $\mathrm{~A}$ \\
\hline $0 €$ & $-150 €$ & $0 €$ & $-75 €$ & A \\
\hline $0 €$ & $-250 €$ & $0 €$ & $-75 €$ & $\mathrm{~A}$ \\
\hline $0 €$ & $-500 €$ & $0 €$ & $-75 €$ & A \\
\hline $0 €$ & $-890 €$ & $0 €$ & $-75 €$ & $\mathrm{~A}$ \\
\hline
\end{tabular}

Table A.9: Series AL2

Consider urn B. In your opinion, what is the probability that the balls in urn B are of the same colour you bet on?

\section{A.2 Use of a Prelec probability weighing function}

In this paper we employ a Tversky and Kahneman (1992) probability weighing function (see Equation (2)). However, another specification can be considered, namely the Prelec (1998) function, which is commonly used in experimental economics. Where $a$ denotes the probability distortion parameters, this function writes as in Equation (14).

$$
f(p)=\exp \left[-(-\ln p)^{a}\right]
$$

We evaluate our results by comparing a Tversky and Kahneman (1992) vs. a Prelec (1998) probability weighing function. This comparison is presented in Table A.10. 


\begin{tabular}{|c|c|c|}
\hline Probability weighing function & Prelec & KT \\
\hline Estimated parameters & Coefficient & Coefficient \\
\hline \multicolumn{3}{|l|}{ Fechner error SD } \\
\hline$\sigma$ & $7.777 * * *$ & $2.158 * * *$ \\
\hline \multicolumn{3}{|l|}{ Risk } \\
\hline$\alpha$ & $0.634 * * *$ & $0.644 * * *$ \\
\hline$\gamma^{+}$ & $0.816^{* * * *}$ & $0.789 * * *$ \\
\hline$\gamma^{-}$ & $0.889 * * *$ & $0.841 * * *$ \\
\hline$\lambda$ & $1.380 * * *$ & $1.361 * * *$ \\
\hline \multicolumn{3}{|l|}{ Ambiguity } \\
\hline$\rho$ & $0.692 * * *$ & $0.722 * * *$ \\
\hline$\phi^{+}$ & $0.508 * * *$ & $0.657 * * *$ \\
\hline$\phi^{-}$ & $0.516 * * *$ & $2.181 * * *$ \\
\hline \#Subjects & 197 & 197 \\
\hline \#Obs & 23,442 & 23,442 \\
\hline Log likelihood & $-12,168.984$ & $-12,090.054$ \\
\hline \multicolumn{3}{|l|}{ Is hypothesis $\mathrm{HO}$ rejected? } \\
\hline \multicolumn{3}{|l|}{ Risk aversion } \\
\hline$\alpha=1$ & Yes*** & Yes*** \\
\hline \multicolumn{3}{|l|}{ Ambiguity aversion } \\
\hline$\rho=1$ & Yes*** & Yes*** \\
\hline \multicolumn{3}{|l|}{ Probability distortion } \\
\hline$\gamma^{+}=1$ & Yes*** & Yes*** \\
\hline$\gamma^{-}=1$ & Yes*** & Yes*** \\
\hline \multicolumn{3}{|l|}{ Distribution distortion } \\
\hline$\phi^{+}=1$ & Yes*** & Yes*** \\
\hline$\phi^{-}=1$ & Yes*** & Yes*** \\
\hline \multicolumn{3}{|l|}{ Gains vs Losses } \\
\hline$\lambda=1$ & Yes*** & Yes*** \\
\hline$\gamma^{+}=\gamma^{-}$ & Yes*** & No \\
\hline$\phi^{+}=\phi^{-}$ & Yes*** & Yes*** \\
\hline \multicolumn{3}{|l|}{ Risk vs Ambiguity } \\
\hline$\alpha=\rho$ & Yes*** & Yes*** \\
\hline$\gamma^{+}=\phi^{+}$ & Yes*** & Yes*** \\
\hline$\gamma^{-}=\phi^{-}$ & Yes*** & Yes*** \\
\hline
\end{tabular}

Table A.10: ML estimations of farmers' risk and ambiguity preferences (197 farmers): Comparing two probability weighing functions.

With the exception of distribution distortion in losses $\phi^{-}$, we find similar results in coefficient magnitudes and signs using a Prelec probability weighing function as we do using a Tversky and Kahneman (1992) probability function. With a Prelec (1998) weighing function, subjects exhibit an inverse $S$-shaped function for distributions in losses. We also find that that probability distortion in risk differs significantly in gains and losses with a Prelec function (highly significant).

\section{A.3 Relaxing the assumption of equality of parameters}

In the regressions in Table 4, we imposed equality of parameters $\alpha^{+}=\alpha^{-}$and equality of parameters $\rho^{+}=\rho^{-}$so that loss aversion is captured by one parameter, $\lambda$. We could also specify a model in which $\lambda=1, \alpha^{+}$and $\alpha^{-}$differ, and $\rho^{+}$and $\rho^{-}$differ. Table A.11 presents the results obtained when the assumption of equality of parameters is imposed versus relaxed. 


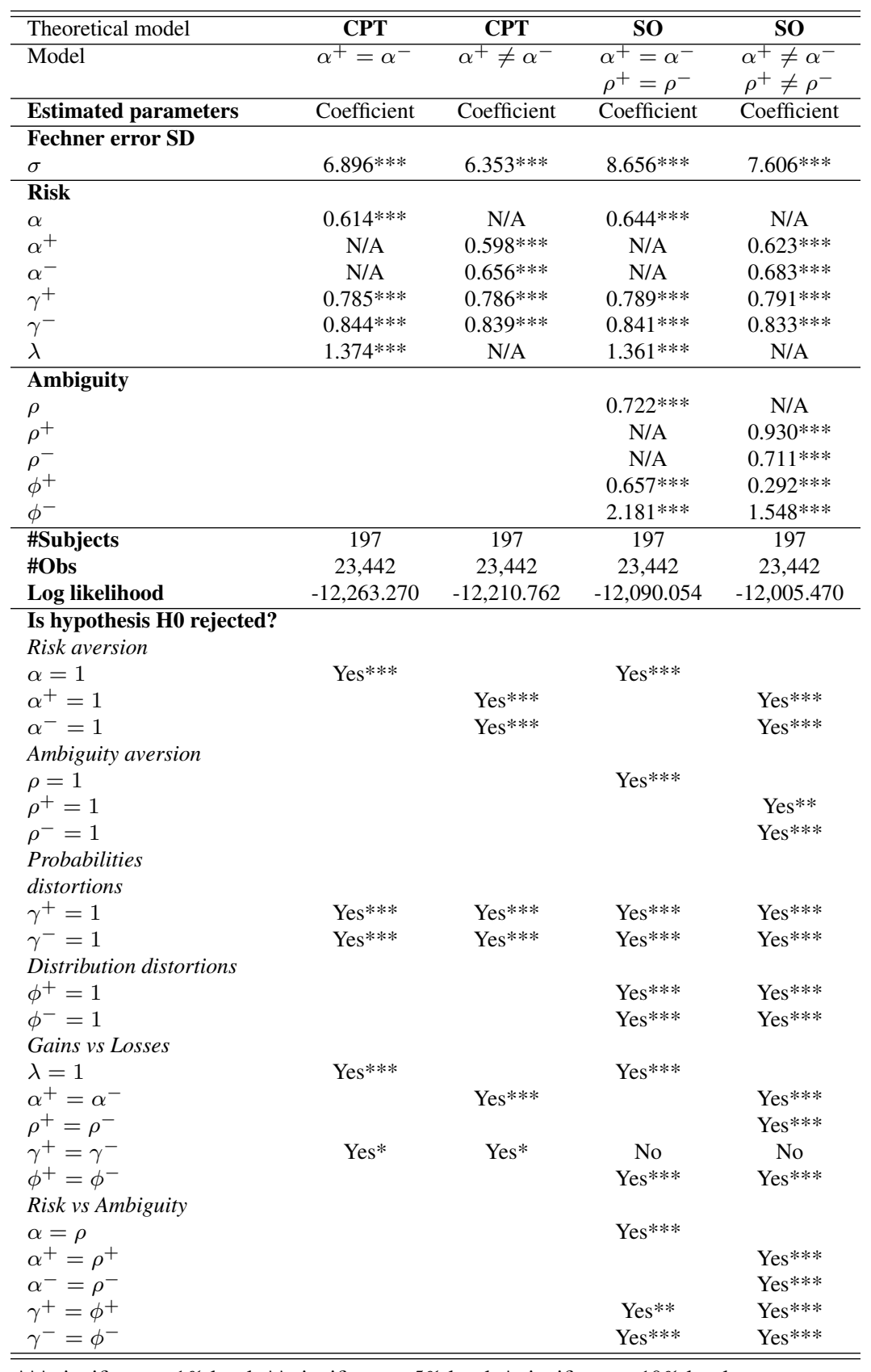

*** significant at $1 \%$ level; ** significant at $5 \%$ level; * significant at $10 \%$ level.

Table A.11: ML estimations of farmers' risk and ambiguity preferences (197 farmers): comparing two parameter assumptions under Cumulative Prospect Theory (CPT) and SecondOrder model (SO).

We find that relaxing the equality of parameters assumption generates similar results to imposing this assumption. We also find evidence of preference reversal: $\alpha^{+} \neq \alpha^{-}$and $\rho^{+} \neq \rho^{-}$(1\% significant).

\section{A.4 Summary information on subjects' lottery choices}

A preliminary step in eliciting farmer preferences is to observe the choices made in these lotteries, specifically the point at which subjects switch from one lottery to the other. We only consider risky lotteries. We do not consider the series on ambiguity because it is difficult to derive ambiguity aversion from observed switching behaviour, as this behaviour is a function of risk preferences. Determining risk aversion, in contrast, is relatively 
straightforward. By observing the number of risky choices (NRCs) made by subjects, we are able to infer subjects' preferences. Specifically, the higher the NRC, the lower the subject's risk aversion. Hellerstein et al. (2013) also study risk preferences among farmers by measuring NRCs and show that this method is a simple way to elicit risk preferences. The advantages of the NRCs method are that no functional form or theory is required and results are independent of any functional form choices. In figure 1 we report the distributions of the number of risky choices made by farmers in the multiple price list series RG1, RG2, RL1 and RL2. Series RG1 and RG2 focus on risk preferences in the gain domain. Series RL1 and RL2 focus on risk preferences in the loss domain. The vertical line in figure 1 represents the number of risky choices maximizing the expected payoff (under risk neutrality). Farmers with NRCs to the right of the vertical line are risk loving, and farmers with NRCs to the left of the vertical line are risk averse.
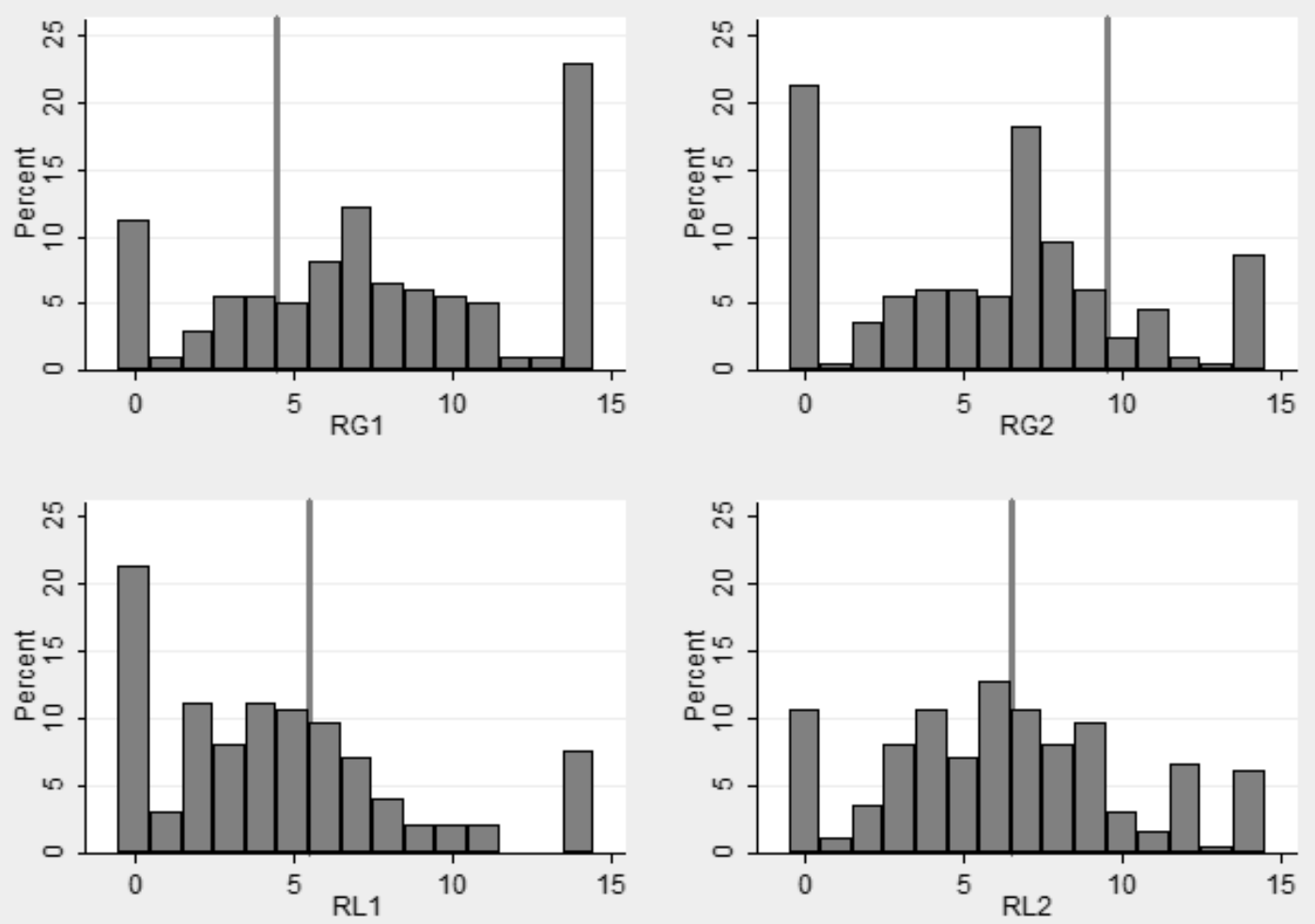

Figure 1: Distribution of the number of risky choices in series RG1, RG2, RL1 and RL2. (Note: The vertical line represents the number of risky choices that maximizes the expected payoff under risk neutrality.)

In figure 1 , we observe that the distributions are not single-spiked as we would have expected if farmer preferences were homogeneous. Specifically, we observe a spike at 0 and a spike at 14, identifying the farmers who always choose the safe option or always choose the risky option, respectively. We also observe a spike at 7 , corresponding to farmers who choose the middle row, which may be driven by a framing effect. The middle row framing effect is neglected in most of the experimental economic studies that employ multiple price list design. In the gain domain, farmers are on average risk seeking in the first series with a mean NRC of 7.70 (s.d. 4.61) and risk averse in the second series with a mean NRC of 5.87 (s.d. 4.24). This result is more striking when considering the simple classification shown in Table A.12.

As we can see in Table A.12, risk preferences in the gain domain are not stable between the first and second series. This could be due to the probability distortions, as the 


\begin{tabular}{l|cc||cc}
\hline Series & \multicolumn{2}{|c}{ Risk averse } & \multicolumn{2}{c}{ Risk seeking } \\
\hline & Whole sample & $\begin{array}{c}\text { Without extreme values } \\
(0 \text { and } 14)\end{array}$ & Whole sample & $\begin{array}{c}\text { Without extreme values } \\
(0 \text { and 14) }\end{array}$ \\
RG1 & $26 \%$ & $23 \%$ & $74 \%$ & $77 \%$ \\
RG2 & $83 \%$ & $88 \%$ & $17 \%$ & $12 \%$ \\
RL1 & $65 \%$ & $62 \%$ & $35 \%$ & $38 \%$ \\
RL2 & $54 \%$ & $52 \%$ & $46 \%$ & $48 \%$ \\
\hline
\end{tabular}

Table A.12: Proportion of farmers according to their preferences and the lottery series

probabilities differ between series, and could cause differences in the evaluation of the lotteries that are not accounted for when using the NRC method.

Another explanation for the differences in elicited preferences between the first and the second series is a possible framing effect, which would increase the tendency for switching at the middle row. As shown in figure 1, the "neutral switching point" is located at row 10 in the first series, which amounts to 4 risky choices (vertical line in series RG1) and occurs below the middle row (7). Every subject who switches at row 10 in this series is considered to be risk seeking. In the second series, the "neutral switching point" occurs at row 4, which corresponds to making 10 risky choices (the vertical line in series RG2), and occurs above the middle row (7). As a result, subjects who switch at row 4 in this series are considered to be risk averse. In the loss domain, farmers are on average risk averse, as NRC means in both series RL1 and RL2 are lower than the NRC of a risk neutral subject. In the first series, mean NRC is 4.55 (s.d. 3.95) and in the second series, mean NRC is 6.31 (s.d. 3.81). In the loss domain, farmer preferences are, on average, stable between the two series.

Using the number of risky choices as a measure of risk preferences enables us to draw several preliminary conclusions regarding these preferences. It also serves as evidence of the extent to which framing effects may be present in our data. This issue is, however, inherent to the multiple price list design. Finally, we note that using the number-of-riskychoice index has certain drawbacks, notably that it lacks precision and captures only a broad estimation of preferences.

\section{A.5 ML estimation of risk preferences under expected utility theory}

Here, we estimate the model under EUT using a constant relative risk aversion (CRRA) utility function, $u(x)=x^{\alpha}$. Parameter $\alpha$ is the curvature parameter that captures risk preferences: $\alpha<1$ for risk aversion, $\alpha=1$ for risk neutrality and $\alpha>1$ for risk seeking. There is only one preference parameter to estimate (along with the Fechner error standard deviation $\sigma$ ). The corresponding results are displayed in Table A.13.

As we aimed to compare the EUT with the estimations performed under the two other, more general, frameworks, all of the choices made by farmers during the field experiment were utilized here, (i.e. those that involved losses and ambiguity). Lottery choices with losses were incorporated using the following transformation of the utility function: if $x<0, u(x)=-(-x)^{\alpha}$. Therefore, in the loss domain, a subject is: risk seeking if $\alpha<1$; risk neutral if $\alpha=1$; and risk averse if $\alpha>1$. Lottery choices with ambiguity were included as follows. Subjects had no information on second-order probabilities in ambiguous lottery series. In order to include these series under EUT, however, we first assumed that these second-order probabilities were known and set them equal to the objective second-order probabilities, i.e., 1/2 in AG1 and AL1 and 1/3 in AG2 and AL2. In other words, we estimated the EUT model as if it were only risk were playing a role. We checked for robustness of our results to the assumption that second-order probabilities 
are known and equal to the objective second-order probabilities. The coefficients change only slightly in magnitude when we use only a subsample of the data from the risk tasks only.

\begin{tabular}{lc}
\hline \hline Estimated parameters & Coefficient \\
\hline Fechner error SD & \\
$\sigma$ & $4.927 * * *$ \\
\hline Risk & $0.574 * * *$ \\
$\alpha$ & 197 \\
\hline \#Subjects & 23,442 \\
\#Obs & $-12,613.746$ \\
\hline Log likelihood & \\
\hline Is hypothesis H0 rejected? & \\
\multicolumn{2}{l}{ Risk aversion } \\
$\alpha=1$ & Yes*** \\
\hline \multicolumn{2}{l}{$* * *$ significant at 1\% level; ** significant at 5\% } \\
level; * significant at 10\% level.
\end{tabular}

Table A.13: ML estimation of farmers' risk preferences under expected utility theory)

The estimated parameter $(\alpha=0.574)$ reveals that farmers are significantly risk averse on average in the gain domain and risk seeking in the loss domain.

\section{A.6 ML estimations of farmers' risk and ambiguity preferences: whole sample vs excluding subjects that do multiple switching}

We compare estimated parameters on the whole sample vs excluding subjects that do multiple switching. Subjects that do multiple switching are defined as having made at least two switchings in at least one of the 9 MPL series. The results are in Table A.14. We find similar overall estimated parameters (only the magnitude slightly differs) except for distribution distorsion in losses $\phi^{-}$. Without MSP (subjects with multiple switching points), subjects have now an inverse S-shaped function for distributions in losses. 


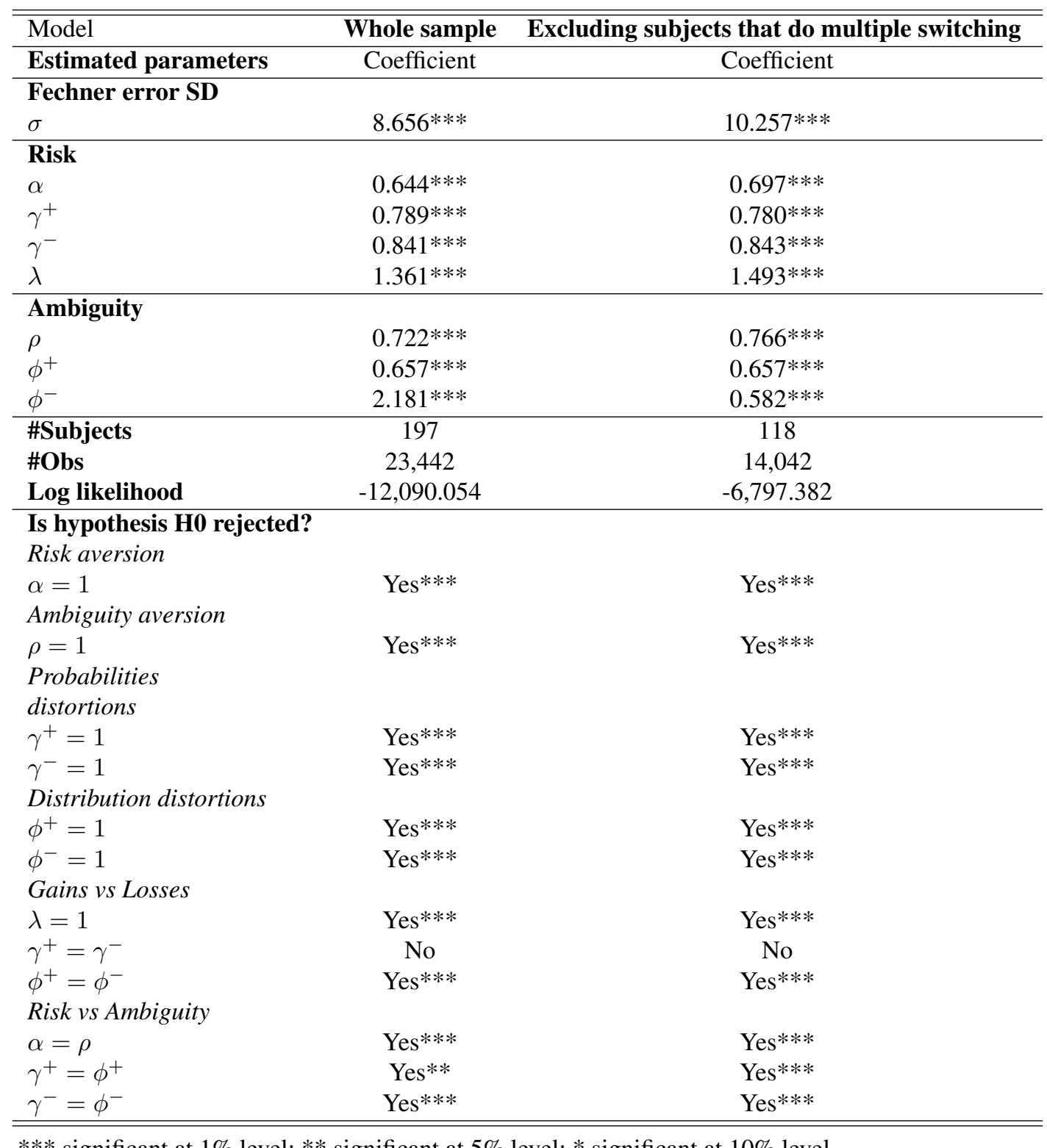

*** significant at $1 \%$ level; ** significant at $5 \%$ level; * significant at $10 \%$ level.

Table A.14: ML estimations of farmers' risk and ambiguity preferences: with and without MSP 